\title{
Numerical Computation of Time Independent and Dependent Dirac Equation Using Atomically Balanced operator and B-spline Basis
}

\author{
by \\ Hebah Rizq, M.Sc. \\ A thesis submitted to the \\ Faculty of Graduate and Postdoctoral Affairs \\ in partial fulfillment of the requirements for the degree of
}

Master of Science in Applied Mathematics

School of Mathematics and Statistics

Ottawa-Carleton Institute of Mathematics and Statistics

Carleton University

Ottawa, Ontario

April, 2014

(C) Copyright

Hebah Rizq, 2014 
The undersigned hereby recommends to the

Faculty of Graduate and Postdoctoral Affairs acceptance of the thesis

\title{
Numerical Computation of Time Independent and Dependent Dirac Equation Using Atomically Balanced operator and B-spline Basis
}

\author{
submitted by Hebah Rizq, M.Sc. \\ in partial fulfillment of the requirements for the degree of \\ Master of Science in Applied Mathematics
}

Professor Dr E. Lorin

Department of School of Mathematics and Statistics

Ottawa-Carleton Institute for Mathematics and Statistics

Department of Mathematics and Statistics

Carleton University

April, 2014 


\section{Abstract}

This thesis is devoted to the numerical computation of the time-independent Dirac equation (TIDE) and the time-dependent Dirac equation (TDDE) in the prolate spheroidal coordinates. Analytical and numerical techniques including Galerkin methods, Min-max principle, and Rayleigh-Ritz methods combined with atomically balanced basis are presented to solve the Dirac equation without spectral pollution. These numerical methods are used to compute the discrete spectrum of the Dirac operator in two-center Coulomb problems for molecules $\mathrm{H}_{2}^{+}$and $\mathrm{Th}_{2}^{179+}$. High order B-spline basis functions are used to obtain accurate results. As excepted, the numerical results do not show any spurious state. 
I dedicate this thesis to my father, Saleh, who passed away in April 7, 2014. 


\section{Acknowledgments}

I would like to express my deepest gratitude to my supervisor Dr E. Lorin for all the guidance, patience, and assistance in my graduate study. Thanks Dr E. Lorin first for accepting me to be one of your students and also for the time which you spent in teaching and encouraging me.

Special thanks to my husband Ahmed, for his remarkable support and wonderful advice which gave me convenience. I owe my loving thanks to my kids Mohamed, Lanah, Feras and Leen for their tolerance and cooperation.

Furthermore, I wish to extend my deepest gratitude to my lovely parents for their encouragement and motivation. Even though, my father is a cancer patient he was calling me daily to ask me about my thesis improvement and praying for me too. Thus he deserves the most thanks and regards. Equally important, my mother she always relief and comfort me with her optimistic words.

My sincere thanks also goes to my government for their generous support both materially and morally. 


\section{Table of Contents}

Abstract $\quad$ iii

Acknowledgments $\quad$ v

Table of Contents $\quad$ vi

List of Tables viii

List of Figures $\quad$ ix

Notations $\quad$ X

1 Introduction $\quad 1$

1.1 Dirac equation ...................... 3

1.2 About spectral pollution . . . . . . . . . . . . . 6

1.3 Organization of the thesis . . . . . . . . . . . 6

2 Numerical computation of the time-independent Dirac equation 7

2.1 Basics on the Dirac operator spectrum . . . . . . . . . . . 8

2.2 Strategies for approximating the Dirac spectrum . . . . . . . . . . . 9

2.3 Existing methods for computing spectra . . . . . . . . . . . . . 10

2.3.1 Galerkin method . . . . . . . . . . . . . . . . 10

2.3.2 Rayleigh-Ritz method ... . . . . . . . . . . . 11

2.3.3 Min-Max principle . . . . . . . . . . . . . . . . 12

2.4 Examples in non-relativistic and relativistic quantum physics . . . . . 14

2.5 Spurious eigenvalue and spurious eigenvectors . . . . . . . . . . 17

2.6 Balanced operator to avoid spectral pollution in Dirac calculation . . 21

2.6.1 Kinetic balance . . . . . . . . . . . . . . . . . . 24 
2.6 .2 Atomic balance . . . . . . . . . . . . . . . . 25

2.7 B-spline . . . . . . . . . . . . . . . . . . . . . . 26

2.7.1 Basic properties of B-splines . . . . . . . . . . 28

2.7.2 Galerkin method with B-spline basis function for timeindependent Dirac equation . . . . . . . . . . . . . . . . 29

3 Numerical results $\quad 35$

3.1 Convergence . . . . . . . . . . . . . . . . . . . . 35

3.2 Full spectrum of diatomic molecules . . . . . . . . . . . . . . 38

4 Time-dependent Dirac equation $\quad 41$

4.1 Discretization of the time-dependent Dirac equation . . . . . . . . . 41

4.2 Mathematical properties . . . . . . . . . . . . . . 46

5 Conclusion $\quad 54$

List of References $\quad 55$ 


\section{List of Tables}

3.1 Results of the numerical computation for the ground state of $\mathrm{H}_{2}^{+}$for different mesh sizes and B-spline of order 7 . Here, $N_{\xi, \eta}$ is the number of elements found in each coordinates, while $N^{*}$ is the total number of the basis functions utilized. The maximum coordinate was fixed to $\xi_{\max }=30 \mathrm{a} . \mathrm{u}$. and the angular momentum to $j_{z}=1 / 2$. The calculations are to be compared with the results from [1], where the authors obtained $E_{\mathrm{H}_{2}^{+}}=-1.10264158103$ a.u. . . . . . . . . . . . .

3.2 Results of the numerical computation for the ground state of $\mathrm{Th}_{2}^{179+}$ for different mesh sizes and B-spline of order 7. $N_{\xi, \eta}$ is the number of elements existing in each coordinates, while $N^{*}$ is the total number of basis functions utilized. The maximum coordinate was fixed to $\xi_{\max }=$ 15 a.u. and the angular momentum to $j_{z}=1 / 2$. The calculations are to be compared with the results from [1] and [2], where the authors obtained $E_{\mathrm{Th}_{179}^{+}}=-9504.77424$ a.u and $E_{\mathrm{Th}_{179}^{+}}=-9504.752$ a.u. . . . .

3.3 Numerical computation of the spectrum of $\mathrm{H}_{2}^{+}$for a mesh size of $30 \times 30$ and B-spline of order 7. The states of the positive and negative continua are computed with the Rayleigh-Ritz, Min-Max principle, and Atomic Balance methods. The first 5 states are shown. . . . . . . . .

3.4 Numerical computation of the spectrum of $\mathrm{Th}_{2}^{179+}$. The mesh size is indicated in the second line and B-splines are of order 7. The atomic

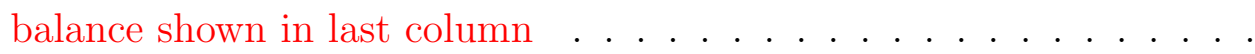




\section{List of Figures}

2.1 The typical spectrum of the non-relativistic Schrödinger operator(left), and the Dirac operator with an external potential (right) [3] . . . . 16

2.2 B-spline of order $3[4] \ldots \ldots$. . . . . . . . . . . . . 27 


\section{Notations}

\begin{tabular}{|c|c|}
\hline$\psi$ & Wavefunction consist of 4-spinors. \\
\hline$\phi$ & Bi-spinors called the large components. \\
\hline$\chi$ & Bi-spinors called the small components. \\
\hline$H_{0}, D^{0}$ & Free Dirac operator. \\
\hline$m$ & Electron mass. \\
\hline$c$ & Light velocity. \\
\hline$V_{c}$ & Coulomb potential. \\
\hline $\mathbf{p}$ & Momentum operator. \\
\hline$\sigma_{i}$ & Pauli matrices \\
\hline$Z_{1,2}$ & Nuclear charges \\
\hline$j_{z}$ & Angular momentum projection on $z$-axis \\
\hline$\nabla$ & Nabla operator. \\
\hline$\triangle$ & Laplace operator. \\
\hline$\lambda, E$ & Eigenvalue, spectral parameter. \\
\hline A & Electromagnetic. \\
\hline$H, F, \mathcal{H}$ & Hilbert space. \\
\hline$B_{i}^{k}(x)$ & B-spline basis functions. \\
\hline$L_{K B}$ & Kinetic Balanced operator. \\
\hline$L_{A B}$ & Atomic Balanced operator. \\
\hline
\end{tabular}




\begin{tabular}{|c|c|}
\hline$P$ & Orthogonal projection. \\
\hline$R$ & The semi-internuclear distance. \\
\hline $\boldsymbol{\alpha}, \alpha_{i}, \beta$ & Dirac matrices. \\
\hline$\alpha$ & $\approx 1 / 137.035999679$ as the fine structure constant. \\
\hline$\sigma(A)$ & Spectrum of Operator $A$. \\
\hline$\sigma_{c}(A)$ & Continuous spectrum of Operator $A$. \\
\hline$\sigma_{d}(A)$ & Discrete spectrum of Operator $A$. \\
\hline$\hbar$ & Planck's constant, equal to 1 in a.u. \\
\hline$\theta$ & Azimuthal angle. \\
\hline$\sigma_{p}(A)$ & The point spectrum of Operator $A$. \\
\hline$\sigma_{r}(A)$ & The residual spectrum of $A$. \\
\hline$\sigma_{e s s}(A)$ & The essential spectrum of Operator $A$. \\
\hline$\langle\cdot \mid \cdot\rangle$ & Hermitian inner product in $\mathbb{R}^{3}$. \\
\hline$H_{2}^{+}$ & Dihydrogen which has $Z_{1,2}=1$. \\
\hline$T h_{2}^{179+}$ & Dithorium which has $Z_{1,2}=90$. \\
\hline$r_{1,2}$ & The distance from nucleus 1 and 2 . \\
\hline
\end{tabular}




\section{Chapter 1}

\section{Introduction}

For the last 10 to 20 years, the numerical computation of the Dirac equation has drawn increasing interest in chemistry and physics. For example, it is now possible to attain laser intensities of up to $10^{23} \mathrm{~W} / \mathrm{cm}^{2}$ [5] in laboratories.

In theory, matter that can undergo such high levels of radiation is supposed to be portrayed in terms of relativistic quantum mechanics and that will call for a solution to the Dirac equation [6]. Relativistic heavy ion collisions has been the most employed framework in the study of the Dirac equation. The main objective is there to find a way to enhance the formation of positron from the collisions of Uranium nuclei [7].

Several methods are aimed at solving the Dirac equation, as it is usually hard to maintain the analytical outlook for solving this equation. Among all the existing methods, one of the most popular ones which has emerged is the operator splitting method. Within this method, the Dirac operator is split into simpler operators. The resultant equations are then more easily solved numerically. By use of this method, the mass potential terms will be considered as a local operator in space and it will be calculated using correct estimates of the time ordered exponential. This method has been employed in [8-11] for the Dirac equation and in $[12,13]$ on the combination of the Maxwell-Dirac equations.

Another possible decomposition of the Dirac Hamiltonian was given in [14-16] using Alternate Direction Iterations. In this case, the spin is kept aligned with the direction of propagation at each step (using a specific rotation in spinor space) such that simple analytical solutions can be found using the method of characteristics. The resulting scheme is also referred to as "Quantum Lattice Boltzmann", which is easy to efficiently parallelize. This method can also modified in the cylindrical coordinate case [17]. While the aforementioned techniques are absolutely efficient, they cannot 
be used to arrive at the original state of the scheme where the confined potential is in states of bound and continuum. From the operator splitting technique, the bound and continuum states are mostly determined from a relativistic variant of the FeitFleck technique $[9,10,17,18]$, which allows to calculate the spectrum and define the bound state wave function from the time-dependent Dirac equation, thus having a very slow convergence.

Other techniques have been developed and the topmost of them all is the mapped Fourier grid. This approach allows easily to evaluate the spectrum and the time evolution of the wave function [19,20]. Direct techniques such as the explicit and implicit finite difference methods that can be found in [21-25].

Techniques for solving the time-independent Dirac equation (TIDE) based on basis set expansion and variational principles were also derived. In this case, variational collapse [26] is one of the main issues. It is related to the fact that the Dirac operator is not bounded from below or above, which induces spurious state in the spectrum. This phenomenon is also called spectral pollution [27]. This thesis is devoted to this kind of method and to spectral pollution problems.

Many techniques have been attempted to circumvent this problem with some measure of good success. The first attempt was the developed of new variational principle, which corresponds to a modification of the usual Rayleigh-Ritz min-max principle, given by Talman in [28] and generalized in [29,30]. However, these methods necessitate solving a nonlinear eigenvalue problem (see [31] for instance). A second method is based on balance principles, which corresponds to a transformation of the basis function expansion [26]. This was first introduced as an empirical rule to get rid of spurious states [32] and was then analyzed by comparing with the non-relativistic results $[27,33,34]$.

There are three usual types of balance approaches: kinetically balanced basis [35], atomically balanced basis [36], and dual kinetically balanced basis [37]. Each of these methods differs in the imposition of the relation between the large and small spinor components.

In this thesis, the atomic balance will be used to calculate the discrete spectrum of the Dirac operator. In this goal a Galerkin method is applied using B-spline basis functions [38-40]. This technique is also used in the framework of heavy ion spectroscopy and heavy ion collision [1,2,19,41-48]. Nonetheless, this is not very well recognized in the dynamic case $[20,25,39]$. The overall objective of this thesis is the 
derivation and analysis of a variational method for TIDE and TDDE using atomic balanced operator and B-spline basis functions.

\subsection{Dirac equation}

The Dirac equation is a quantum wave equation, which describes the relativistic dynamics of spin- $\frac{1}{2}$ particles (fermions), such as electrons. The time independent Dirac equation (eigenvalue problem) is given by:

$$
H_{0} \psi(x)=E \psi(x) \text { with } H_{0} \equiv c \boldsymbol{\alpha} \cdot \mathbf{p}+m c^{2} \beta+V_{c}(x) \mathbb{I}_{4},
$$

The particle under consideration is characterized by a 4 -spinor,

$$
\psi=[\phi, \chi]^{T} \in C^{1}\left(0, T ; L^{2}\left(\mathbb{R}^{3}, \mathbb{C}^{4}\right)\right)
$$

with positive time $T$. The 2-spinors $[\phi, \chi] \in C^{1}\left(0, T ; L^{2}\left(\mathbb{R}^{3}, \mathbb{C}^{2}\right)\right)$ where $\phi$ is the large component, and $\chi$ is the small one.

The dynamics of the wave function, (time-dependent case) is given by the following equation:

$$
\mathrm{i} \partial_{t} \psi=H_{0} \psi, \text { with } H_{0} \equiv c \boldsymbol{\alpha} \cdot \mathbf{p}+m c^{2} \beta+V_{c}(x) \mathbb{I}_{4},
$$

where $H_{0}$ is again the Hamiltonian operator, $i$ imaginary unit, $\boldsymbol{\alpha}$ and $\beta$ are the Dirac matrices, $V_{c}$ is the Coulomb potential, $\mathbf{p}=-i \boldsymbol{\nabla}$ is the momentum operator, $c$ is the light velocity, $m$ is the electron mass, and $\psi$ is the four component spinor.

Note that all calculations will be performed in atomic units (a.u.), where $m=1, \hbar=1$

and $c=1 / \alpha$ where we consider $\alpha \approx 1 / 137.035999679$ the constant of fine structure. However, the mass is explicitly kept during all the equations, thus allowing for a smooth conversion from atomic to natural units. The matrix structure is given by $\boldsymbol{\alpha}$ and $\beta$ in $M_{4}(\mathbb{C})$ :

$$
\boldsymbol{\alpha}=\left[\begin{array}{cc}
0 & \sigma_{i} \\
\sigma_{i} & 0
\end{array}\right] \text { and } \beta=\left[\begin{array}{cc}
\mathbb{I}_{2} & 0 \\
0 & -\mathbb{I}_{2}
\end{array}\right]
$$


where $\sigma_{i}=\left(\sigma_{x}, \sigma_{y}, \sigma_{z}\right)$ are the Pauli matrices. The latter are

$$
\sigma_{x}=\left[\begin{array}{cc}
0 & 1 \\
1 & 0
\end{array}\right], \sigma_{y}=\left[\begin{array}{cc}
0 & -i \\
i & 0
\end{array}\right] \text { and } \sigma_{z}=\left[\begin{array}{cc}
1 & 0 \\
0 & -1
\end{array}\right] \text {. }
$$

In time dependent Dirac equation (TDDE) a relativistic spin- $\frac{1}{2}$ quantum particle is a subject to a classical electromagnetic field $(\mathbf{A}, V) \in C^{2}\left(\mathbb{R}^{3} \times \mathbb{R}_{+}, \mathbb{R}^{3} \times \mathbb{R}_{+}\right)$. First, we will start by assuming that the electromagnetic field is known at any given time and that the back-reaction of the particle on the electromagnetic field is negligible. The equation under consideration then is:

$$
\mathrm{i} \partial_{t} \psi=H \psi, H=\boldsymbol{\alpha} \cdot(-\mathrm{i} c \nabla-e \mathbf{A})+m c^{2} \beta+\left(V_{c}(x)+V(x, t)\right) \mathbb{I}_{4},
$$

where the electromagnetic field is added by the minimal coupling prescription. This technique guarantees a gauge-invariant formulation. However, in explicit calculations, a specific gauge is rather preferred; the Coulomb gauge is chosen where $V=0$, in which case, the existence of an electric charge in $V_{c}$ may be characterized by a Coulomb potential see [49]. The latest equation offers a consistent description of bound electrons in molecules in the Born-Oppenheimer approximation, (nuclei are fixed in space and included in the potential term $V_{c}$ ). This becomes a valid approximation when the mass of the nucleus is quite larger than the mass of the electron, which is always the situation for the systems considered in this study. As aforementioned, an interesting system is the two-center system where two nuclei described by the Coulomb potential are considered, such as:

$$
V_{c}(x, y, z)=-\frac{Z_{1} e}{\sqrt{x^{2}+y^{2}+(z-R)^{2}}}-\frac{Z_{2} e}{\sqrt{x^{2}+y^{2}+(z+R)^{2}}},
$$

where $Z_{1,2}$ are the nuclear charges and $R$ is the semi-internuclear distance. To solve this system, firstly, it is preferred to consider cylindrical coordinates where

$$
\begin{aligned}
& x=r \cos (\theta), \\
& y=r \sin (\theta),
\end{aligned}
$$

with $r=\sqrt{x^{2}+y^{2}}$ taken as the radial distance from nuclei and $\theta=\tan ^{-1}(y / x)$ the azimuthal angle. Assume that the Dirac equation has azimuthal symmetry, means 
that the electrodynamic potential does not depend on $\theta$. In that case the number of dimensions is reduced from 3 to 2 , using separation of variables. The $\theta$-dependence can be included using the following ansatz applied to the four-spinor with cylindrical symmetry $[1,50]$ :

$$
\Psi(\mathbf{x}, t)=\left[\begin{array}{c}
\psi_{1}(t, r, z) e^{i \mu_{1} \theta} \\
\psi_{2}(t, r, z) e^{i \mu_{2} \theta} \\
\psi_{3}(t, r, z) e^{i \mu_{1} \theta} \\
\psi_{4}(t, r, z) e^{i \mu_{2} \theta}
\end{array}\right]
$$

where $\mu_{1,2}:=j_{z} \mp 1 / 2$ and where $j_{z}$ is the angular momentum projection on the $z$-axis (it can take one of the values $j_{z}=\left(\cdots,-\frac{5}{2},-\frac{3}{2},-\frac{1}{2}, \frac{1}{2}, \frac{3}{2}, \frac{5}{2}, \cdots\right)$. Substituting in the Dirac equation leads to

$$
\begin{aligned}
i \partial_{t} \psi(t, r, z)= & \left\{\alpha_{x}\left[-i c \partial_{r}-i c \frac{1}{2 r}-e A_{r}(t, r, z)\right]+\alpha_{y}\left[c \frac{j_{z}}{r}-e A_{\theta}(t, r, z)\right]\right. \\
& \left.+\alpha_{z}\left[-i c \partial_{z}-e A_{z}(t, r, z)\right]+\beta m c^{2}+e V_{c}(r, z)\right\} \psi(t, r, z) .
\end{aligned}
$$

The prolate spheroidal coordinates $\xi$ and $\eta$ are related to the cylindrical coordinates $r$ and $z$ as follows:

$$
\begin{aligned}
& r=R\left[\left(\xi^{2}-1\right)\left(1-\eta^{2}\right)\right]^{\frac{1}{2}} \\
& z=R \xi \eta
\end{aligned}
$$

where $\xi \in[1, \infty), \eta \in[-1,1]$ and $\theta=[0,2 \pi]$ (azimuthal angle). In order to obtain the Dirac equation in these coordinates; one simply can use the mapping in Eqs.(1.11) and (1.12) along with the derivatives

$$
\left\{\begin{array}{l}
\partial_{r}=\frac{\sqrt{\left(\xi^{2}-1\right)\left(1-\eta^{2}\right)}}{R\left(\xi^{2}-\eta^{2}\right)}\left[\xi \partial_{\xi}-\eta \partial_{\eta}\right] \\
\partial_{z}=\frac{\left(\xi^{2}-1\right)}{R\left(\xi^{2}-\eta^{2}\right)} \eta \partial_{\xi}+\frac{\left(1-\eta^{2}\right)}{R\left(\xi^{2}-\eta^{2}\right)} \xi \partial_{\eta}
\end{array}\right.
$$

The prolate spheroidal coordinates yield more accurate results in both relativistic $[1,44]$ and non-relativistic cases [51-53]. Moreover, within these coordinates, the nuclei are positioned at the corners of the domain, thus facilitating the numerical 
implementation. Consequently, these coordinates will be used throughtout this work.

\subsection{About spectral pollution}

Spectral pollution is a significant issue, which can be found in many different practical situations. For instance, it is encountered when dealing with the Schrödinger operator $[54,55]$, as in elasticity, electromagnetism and hydrodynamics [56-62].

This phenomenon happens when one approximates the spectrum of a (bounded or unbounded) self-adjoint operator $A$ on an infinite-dimensional Hilbert space, using a sequence of finite-dimensional spaces. More precisely, we approximate the operator $A$ by $A_{n}$ such that " $\lim _{n \rightarrow \infty} A_{n}=A^{\prime \prime}$ (here, $n$ is the dimension of the subspace on which the operator is projected or, loosely speaking, the dimension of the operator matrix once the problem is discretized). The discretized operator $A_{n}$ has eigenvalues given by $\lambda_{n} \in \sigma_{A_{n}}$. The set of spurious states $\sigma_{A_{n}}^{s} \subset \sigma_{A_{n}}$ is defined by the set of all eigenvalues in $\sigma_{A_{n}}$ for which $\lim _{n \rightarrow \infty} \lambda_{n} \notin \sigma_{A}$ [43].

The spectrum of the free Dirac operator is $\left(-\infty,-m c^{2}\right] \cup\left[m c^{2}, \infty\right)$. Adding potential usually creates eigenvalues in the gap $\left(m c^{2},-m c^{2}\right)[3,27]$. In Section 2.6, we will illustrate some general properties about spectral pollution. We refer to [3] for more details.

\subsection{Organization of the thesis}

The outline of this thesis is as follows. In Chapter 2, we present the numerical computation of the time-independent Dirac equation (TIDE) using atomic balanced operator and $B$-spline basis functions to compute the relativistic spectra of two center problems. In Chapter 2 spectral pollution and basic facts about the Dirac operator spectrum are presented. In addition, some examples in non-relativistic and relativistic quantum physics, and fundamental properties of $B$-spline basis functions are discussed. Chapter 3 is devoted to numerical results for two-center Coulomb problem: diatomic molecule $\mathrm{H}_{2}^{+}$and $\mathrm{Th}_{2}^{179+}$. The discretization of the time-dependent Dirac equation (TDDE) with some mathematical properties of the derived schemes is presented in Chapter 4. We conclude in Chapter 5. 


\section{Chapter 2}

\section{Numerical computation of the time-independent Dirac equation}

In this chapter we present a numerical method to compute the spectrum of the timeindependent Dirac equation. This equation writes as follows:

$$
H_{0} \psi(x)=E \psi(x)
$$

where $\psi=[\phi, \chi]^{T}$, where $\phi$ is refer to the large component and $\chi$ is the small component. $H_{0} \equiv c \boldsymbol{\alpha} \cdot \mathbf{p}+m c^{2} \beta+V_{c}(x) \mathbb{I}_{4}$ is the Dirac Hamiltonian operator. $\mathbf{p}=-i \boldsymbol{\nabla}$ is the momentum operator, $c$ is the light velocity, $m$ is the electron mass, $E$ is the electron energy and $\psi \in L^{2}\left(\mathbb{R}^{3}, \mathbb{C}^{4}\right)$ is a four component spinor. $\boldsymbol{\alpha}=\left(\alpha_{x}, \alpha_{y}, \alpha_{z}\right)$ are the Dirac matrices, and the matrix structure is given by $\boldsymbol{\alpha}$ and $\beta$ in $M_{4}(\mathbb{C})$ :

$$
\alpha_{i}=\left[\begin{array}{cc}
0 & \sigma_{i} \\
\sigma_{i} & 0
\end{array}\right] \text { and } \beta=\left[\begin{array}{cc}
\mathbb{I}_{2} & 0 \\
0 & -\mathbb{I}_{2}
\end{array}\right] \text {. }
$$

where $\sigma_{i}=\left(\sigma_{x}, \sigma_{y}, \sigma_{z}\right)$

$$
\sigma_{x}=\left[\begin{array}{ll}
0 & 1 \\
1 & 0
\end{array}\right], \sigma_{y}=\left[\begin{array}{cc}
0 & -i \\
i & 0
\end{array}\right] \text { and } \sigma_{z}=\left[\begin{array}{cc}
1 & 0 \\
0 & -1
\end{array}\right] \text {. }
$$

Through this chapter, spectral pollution will also be presented with strategies to avoid it. Basic properties, definitions, and simple examples will be discussed. 


\subsection{Basics on the Dirac operator spectrum}

We introduce some basic definitions in spectral theory before discussing the numerical computation of TIDE [49,63].

Let $A$ be an unbounded operator on a complex Banach space $X$, with domain $D(A)$. The resolvant of $A$, at $\lambda$, denoted by $\rho(A)$ and defined as the inverse of $(A-\lambda \mathbb{I})$, where $\lambda \in \mathbb{C}$ and $\mathbb{I}$ is the identity on $X$

$$
R(\lambda, A)=A_{\lambda}^{-1}=(A-\lambda \mathbb{I})^{-1}
$$

We denote by $\sigma(A)$ the spectrum of $A$, which is the complement in $\mathbb{C}$ of the resolvant set $\rho(A)$. We then have

$$
\begin{gathered}
\sigma(A) \cup \rho(A)=\mathbb{C} . \\
\sigma(A) \cap \rho(A)=\emptyset .
\end{gathered}
$$

Definition 2.1.1 Note that $\sigma(A)$ is the union of three disjoint sets, denoted respectively by $\sigma_{p}(A)$ the point spectrum of Operator $A, \sigma_{c}(A)$ the continuous spectrum of $A$, and $\sigma_{r}(A)$ the residual spectrum of $A$.

1. $\sigma_{p}(A)$ is the set of all points $\lambda \in \mathbb{C}$ for which $A_{\lambda}$ is non-invertible.

2. $\sigma_{c}(A)$ is the set of all points $\lambda \in \mathbb{C}$ for which $A_{\lambda}^{-1}$ is unbounded on $X$, with domain dense in $X$.

3. $\sigma_{r}(A)$ is the set of all points $\lambda \in \mathbb{C}$ for which $A_{\lambda}^{-1}$ exists, with domain not dense in $X$.

The discrete spectrum and the essential spectrum are defined respectively as follows [63]:

1. $\sigma_{d}(A)$ is the set of all eigenvalues of Operator $A$ with finite multiplicity, which are isolated points of spectrum.

2. $\sigma_{\text {ess }}(A)$ is the complement in $\mathbb{C}$ of the discrete spectrum, that is $\sigma_{e s s}(A) \equiv \sigma(A) \backslash \sigma_{d}(A)$.

We refer to [49] for more details. 


\subsection{Strategies for approximating the Dirac spec- trum}

The process for approximating the eigenvalues of the Dirac operator is as follows [3]:

$$
D^{V}:=D^{0}+V(r)
$$

where $D^{V}$ Dirac operator with electrostatic potential, $D^{0}$ is the free Dirac operator also denoted $H_{0}$ and defined as,

$$
D^{0}=c \alpha \cdot(-i \nabla)+m c^{2} \beta
$$

and $V(r)$ electrostatic potential. To solve the eigenvalue equation

$$
\left(D^{V}\right)_{\mid W} x=\lambda S x
$$

$W$ is chosen as a $d$-dimensional vector space, such that $W \subseteq D\left(D^{0}+V\right)=H^{1}\left(\mathbb{R}^{3}\right)$ and $\left(D^{V}\right)_{\mid W}$ is the restriction matrix of $D^{V}$ to $W$. Denoting by $\left\{b_{1}(r), \cdots, b_{d}(r)\right\}$ a basis of $W$, the associated $d \times d$ matrix is $\left(D^{V}\right)_{\mid W}=\left(\left\langle b_{i}, D^{V} b_{j}\right\rangle\right)_{1 \leq i, j \leq d}$. The matrix $S=\left(\left\langle b_{i}, b_{j}\right\rangle\right)_{1 \leq i, j \leq d}$ is the overlap matrix. The inner product for 4 -spinors is defined by

$$
\langle\psi, \phi\rangle=\int_{\mathbb{R}^{3}} \psi(r)^{*} \phi(r) d^{3} r=\sum_{j=1}^{4} \int_{\mathbb{R}^{3}} \psi(r)_{j}^{*} \phi(r)_{j}^{*} d^{3} r .
$$

The expectation is that the spectrum of Matrix $\left(D^{V}\right)_{\mid W}$ is a good approximation of the elements of the spectrum of $D^{V}$. Approximation must be improved as the size of the basis grows. Therefore, it is usually considered a sequence of discretization spaces $W_{n}$ such as $\operatorname{dim} W_{n} \rightarrow \infty$, and the approximate eigenvalues converge to the true ones as $n \rightarrow \infty$, expecting also that, for any $\psi \in H^{1}\left(\mathbb{R}^{3}, \mathbb{C}^{4}\right)$, there exists an approximate sequence $\psi_{n} \in W_{n}$ with

$$
\lim _{n \rightarrow \infty} \int_{\mathbb{R}^{3}}\left(\left|\psi_{n}(r)-\psi(r)\right|^{2}+\left|\nabla\left(\psi_{n}-\psi\right)(r)\right|^{2}\right) d^{3} r=0
$$

This completeness condition is satisfied for most approximation schemes. We refer to [3] for more discussion about approximating eigenvalues and eigenfunctions. 


\subsection{Existing methods for computing spectra}

We describe and discuss some of the most important methods used to calculate the spectrum of time-independent Dirac equation.

\subsubsection{Galerkin method}

The Galerkin Method is used to approximate the eigenvalue problem in a finitedimensional space [64,65].

$$
H(u, v)=\lambda(u, v), \quad \forall v \in V
$$

where $H$ is a self-adjoint operator on a Hilbert space $V$, and $\lambda$ is a (real) eigenvalue of $H$.

The Galerkin method consists of approximating the weak formulation (2.6) by

$$
H\left(u_{h}, v_{h}\right)=\lambda\left(u_{h}, v_{h}\right) \quad \forall v_{h} \in V_{h} .
$$

where $V_{h}$ is a finite dimensional vector subspace of $V$ of dimension $N$, with a basis $\left\{\phi_{1}, \cdots, \phi_{N}\right\}$. Then

$$
u_{h}(x)=\sum_{j=1}^{N} u_{j} \phi_{j}(x)
$$

To determine the unknown coefficient $u_{j}$, we multiply both sides of $(2.7)$ by test functions $v_{h}=\phi_{i}$,

$$
\sum_{j=1}^{N}\left(H u_{j} \phi_{j}, \phi_{i}\right)=\lambda \sum_{j=1}^{N} u_{j}\left(\phi_{j}, \phi_{i}\right) \quad \forall i=1, \cdots, N
$$

Introducing a matrix $A=\left(a_{i j}\right)_{i j}$ where $a_{i j}=\left(H \phi_{j}, \phi_{i}\right)$, we write $(2.8)$ as:

$$
A U=\lambda U
$$

where $U$ is the vector $\left[u_{1}, \cdots, u_{N}\right]^{T}$. The structure of the matrix $A$, as well as the degree of accuracy, depends on the chosen basis function $\left\{\phi_{i}\right\}$, and on the choice of $V_{h}[64]$. 


\subsubsection{Rayleigh-Ritz method}

The Rayleigh-Ritz method relies on a variational principle, which enables the estimation of the eigenvalues of an operator on a Hilbert space. This method has been extensively used in relativistic and non-relativistic problems, see for instance [66]. The steps of solving the eigenvalue problem in the Dirac equation are as follows:

$$
H_{0} \psi=E \psi
$$

Both sides of (2.10) are then multiplied by $\psi=[\phi, \chi]^{T}$ and integrated. The variational formulation leads then to find stationary points of the functional

$$
\begin{array}{r}
\int_{\mathbb{R}^{3}}\left[m c^{2}+V\right]|\phi|^{2}+\left\langle\phi \mid R_{0} \chi\right\rangle_{L^{2}\left(\mathbb{R}^{3}, \mathbb{C}^{2}\right)}+\left\langle\chi \mid R_{0} \phi\right\rangle_{L^{2}\left(\mathbb{R}^{3}, \mathbb{C}^{2}\right)}+\left[V-m c^{2}\right]|\chi|^{2}= \\
E \int_{\mathbb{R}^{3}}|\phi|^{2}+|\chi|^{2} .
\end{array}
$$

The last formula is an explicit instance of the Rayleigh-Ritz coefficient [43]:

$$
\bar{H}_{0}=\frac{\left\langle\psi\left|H_{0}\right| \psi\right\rangle_{L^{2}\left(\mathbb{R}^{3}, \mathbb{C}^{4}\right)}}{\langle\psi \mid \psi\rangle_{L^{2}\left(\mathbb{R}^{3}, \mathbb{C}^{4}\right)}}
$$

The notation $\langle\cdot \mid \cdot\rangle$ refers to the Hermitian inner product on $L^{2}\left(\mathbb{R}^{3}, \mathbb{C}^{4}\right)$. We introduce now two operators $C$ and $S$ by:

$$
\begin{aligned}
C[\psi] & \left.=\int_{\mathbb{R}^{3}}\left[m c^{2}+V_{c}\right]|\phi|^{2}+\right\rangle R_{0} \phi|\chi\langle+\rangle \chi| R_{0} \phi\left\langle+\left[V_{c}-m c^{2}\right]|\chi|^{2} .\right. \\
S[\psi] & =\int_{\mathbb{R}^{3}}|\phi|^{2}+|\chi|^{2} .
\end{aligned}
$$

It is well known that the convergence of the Rayleigh-Ritz method depends mainly on the structure of the operator spectrum. For the Dirac operator, the spectrum is not bounded and the quantity $\bar{H}_{0}$ does not form an upper or lower bound. The eigenvalues can however be characterized by a min-max principle, which is discussed in Section 2.3.3. The convergence of this approach is not guaranteed, due to the fact that the stationary point is a saddle point, and spurious states may then appear [43]. As a result, a modification of this method is required to improve the convergence. A numerical scheme can be derived from these equations by discretization of the eigenvalue problem, over a set of $B$-spline basis functions. The strategy used in this 
thesis is to use Atomically Balanced Basis Functions (ABBF). Finding the eigenvalues of minimization procedure in Eq. (2.12) is similar to finding the stationary point of the functional.

$$
\mathcal{E}[\psi]=\left\langle\psi\left|H_{0}\right| \psi\right\rangle_{L^{2}\left(\mathbb{R}^{3}, \mathbb{C}^{4}\right)}-E\langle\psi \mid \psi\rangle_{L^{2}\left(\mathbb{R}^{3}, \mathbb{C}^{4}\right)},
$$

where $E$ is a Lagrange multiplier. This form is used in the following to convert the basis set expansion into a generalized eigenvalue problem.

\subsubsection{Min-Max principle}

The min-max method was developed by Talman [28] and Datta-Deviah [67] for the case of the Dirac operator with a Coulomb potential. The convergence and accuracy of this method was then studied by Esteban [68]. The main idea of this method is to find the stationary points of the Rayleigh-Ritz coefficient as follows:

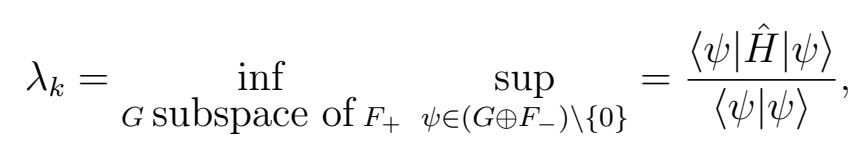

where the dimension of $G$ is $k$, and $F_{+}, F_{-}$are two orthogonal subspaces of Hilbert space $F \subseteq L^{2}\left(\mathbb{R}^{3}, \mathbb{C}^{4}\right)$. For practical purpose, it is convenient to use one of these decompositions and the large and small components of the Dirac equation as [30]:

$$
F_{+}=L^{2}\left(\mathbb{R}^{3}, \mathbb{C}^{2}\right) \otimes[0,0]^{T}, \quad F_{-}=[0,0]^{T} \otimes L^{2}\left(\mathbb{R}^{3}, \mathbb{C}^{2}\right)
$$

The maximization procedure stems from the relation between the large $\phi$ and small $\chi$ components required to solve the Dirac equation.

The four-spinors can be written as two bi-spinors $\psi \equiv[\phi, \chi]^{T}$ where $\phi$ is the large component, and $\chi$ is the small one as defined in. The Dirac equation (2.10) becomes:

$$
\left[\begin{array}{cc}
V_{c}(x)+m c^{2} & R_{0} \\
R_{0} & V_{c}(x)-m c^{2}
\end{array}\right]\left[\begin{array}{l}
\phi(x) \\
\chi(x)
\end{array}\right]=E\left[\begin{array}{l}
\phi(x) \\
\chi(x)
\end{array}\right],
$$

where we define $R_{0}=(-i c \boldsymbol{\sigma} \cdot \nabla)$. 
Equation (2.17) is rewritten as:

$$
\left\{\begin{array}{l}
\left(V_{c}(x)+m c^{2}\right) \phi(x)+R_{0} \chi(x)=E \phi(x) \\
R_{0} \phi(x)+\chi(x)\left(V_{c}(x)-m c^{2}\right)=E \chi(x)
\end{array}\right.
$$

then as

$$
\left\{\begin{array}{l}
R_{0} \chi(x)=\left[E-m c^{2}-V_{c}(x)\right] \phi(x) \\
R_{0} \phi(x)=\left[E+m c^{2}-V_{c}(x)\right] \chi(x) .
\end{array}\right.
$$

The small component $\chi$ can then be written in terms of the large component $\phi$ as

$$
\chi(x)=\frac{R_{0}}{E+m c^{2}-V_{c}(x)} \phi(x)
$$

by substitution $(2.20)$ in $(2.19)$, we get

$$
R_{0}\left[\frac{R_{0} \phi(x)}{E+m c^{2}-V_{c}(x)}\right]=\left[E-m c^{2}-V_{c}(x)\right] \phi(x) .
$$

The last equation can be observed, by minimizing the energy over any couple $A[E, \phi]$ defined below. Then we multiply $(2.21)$ by a large component $\phi \in\left(\mathbb{R}^{3}, \mathbb{C}^{2}\right)$ on the left, integrate by parts and then use the divergence theorem. The functional equation is then given by:

$$
A[E, \phi]=\int d^{3} x\left[\left(\frac{|\hat{R}|^{2}}{E+m c^{2}-V}\right)-\left[E-m c^{2}-V\right]|\phi|^{2}\right]=0 .
$$

The wave function should vanish faster than $\sim \frac{1}{r^{2}}$ at infinity. In the Coulomb potential case the corresponding wave function vanishes as $\phi \sim e^{-r}$ when $r \rightarrow \infty$ [69]. As a consequence, (2.22) offers a realization of the min-max principle. Moreover, the discretization without variational collapse would indicate that the calculated energy spectrum is bounded in the mass gap. Note that, the spurious states do not appear in the calculated discrete spectrum. We refer to [43] for more details about min-max method. 


\subsection{Examples in non-relativistic and relativistic quantum physics}

In this section we recall some necessary results and definitions about the spectrum of the (non-relativistic) Schrödinger operator, as well as the (relativistic) Dirac operator $[3,27]$.

Definition 2.4.1 An operator $A$ is said self-adjoint on a Hilbert space $H$ with domain $D(A)$, if $A=A^{*}$, that is if $\langle A x, y\rangle=\langle x, A y\rangle$ for all $x, y \in D(A)$.

We now list a few properties about the Dirac operator with Coulomb potential [70].

Remark 2.4.1 An electron in the field of a point nucleus is described by the Coulomb potential [70]:

$$
\phi_{e l}(x)=\frac{\gamma}{|x|}, \text { and, } \gamma=\frac{e^{2} Z}{\hbar} \text {, where } Z \text { is the nuclear charge, }
$$

For coupling constants $|\gamma|<c / 2(Z \leq 68)$ the Coulomb potential is covered by the assumptions:

$$
\left|V_{i k}(x)\right| \leq a \frac{c}{2|x|}+b, \forall x \in \mathbb{R}^{3} \backslash\{O\}, \quad i, k=1 \cdots, 4
$$

where $\hbar=1$ and $c=1 / \alpha$ where we consider $\alpha \approx 1 / 137.035999679$. Also, a, $b$ are constants such that $b>0$, and $a<1$. Operator $H=H_{0}+V(x)$, where $H_{0}$ is the free Dirac operator defined in Section 1.1, is essentially self-adjoint on $\mathcal{C}_{0}^{\infty}\left(\mathbb{R}^{3} \backslash\{O\}\right)^{4}$ and self-adjoint on $D\left(H_{0}\right)=H^{1}\left(\mathbb{R}^{3}\right)^{4}$. The restriction on $\gamma$ is quite unfamiliar from the nonrelativistic theory. The essential self-adjointness indeed breaks down, and is a technical constraint if $|\gamma|>c \sqrt{3} / 2(Z>118)$.

We now state two important theorems about the domain of the Dirac operator in the case of a Coulomb potential [70]

Theorem 2.4.1 Operator $H$ is essentialy self-adjoint if and only if for some $\lambda \in \mathbb{C}$ the equation $H f=\lambda f$ has a solution $f \notin L^{2}(0, R)^{2}$ for some $R>0$, i.e, a solution which is not square integrable at the origin. 
Theorem 2.4.2 Assume that the electrostatic potential $V=\phi_{\text {el }} \mathbf{1}$ where $\phi_{\text {el }}$ defined in Eq. (2.23) satisfies the condition

$$
\sup _{x \in \mathbb{R}^{3} \backslash\{0\}}\left|x \phi_{e l}(x)\right|<\gamma
$$

Then for $\gamma \leq \sqrt{3} / 2$ the Dirac operator $H=H_{0}+\phi_{e l} \mathbf{1}$ is essentially self-adjoint on $D=C_{0}^{\infty}\left(\mathbb{R}^{3} \backslash\{0\}\right)$ and self-adjoint on $D(H)=D\left(H_{0}\right)$. For $\gamma<c$ the Dirac operator $H$, defined on $\mathcal{D}$, has a self-adjoint extension $\bar{H}$ which is uniquely characterized by the property

$$
D(\bar{H}) \subset D\left(\left|H_{0}\right|^{1 / 2}\right) \quad \text { or equivalently } \quad D(\bar{H}) \subset D\left(|V|^{1 / 2}\right) .
$$

The following definition is about Kato potential (see [63] for more details).

Definition 2.4.2 A potential function $V$ is called a Kato potential, if it is real and $V \in L^{2}\left(\mathbb{R}^{n}\right)+L^{\infty}\left(\mathbb{R}^{n}\right)_{\epsilon}$, where $\epsilon>0, V=V_{1}+V_{2}$ with $V_{1} \in L^{2}\left(\mathbb{R}^{n}\right)$ and $V_{2} \in L^{\infty}\left(\mathbb{R}^{n}\right)$, such that $\left\|V_{2}\right\|_{\infty}<\epsilon$.

Example 1 In the non-relativistic case, consider the operator $A=\frac{-\hbar^{2} \triangle}{2 m}$ defined on the Hilbert space $H=L^{2}\left(\mathbb{R}^{3}\right)$, where $\triangle$ is a Laplace operator. To ensure that Operator $A$ is a map function in the domain $D\left(\frac{-\hbar^{2} \triangle}{2 m}\right)$, we must assume that $\triangle \psi$ is a square integrable function on $\mathbb{R}^{3}$, that is $D\left(\frac{-\hbar^{2} \triangle}{2 m}\right)=H^{2}\left(\mathbb{R}^{3}, \mathbb{C}\right)$ as follows:

The spectrum of the self-adjoint operator $\triangle$ on $H^{2}\left(\mathbb{R}^{n}\right)$ is $\sigma(-\triangle)=\sigma_{\text {ess }}(-\triangle)=$ $[0, \infty)$ according to Theorem (7.6) in [49], Then

$$
\operatorname{Spec}\left(-\hbar^{2} \triangle / 2 m\right)=[0, \infty)
$$

There is no eigenvalue in this spectrum i.e. there does not exist any square integrable $\psi$ such that

$$
\left(\frac{-\hbar^{2} \triangle}{2 m}\right) \psi=\lambda \psi
$$

There only exist approximate $\psi_{n}$, and $\lambda_{n}$ which satisfy $\int_{\mathbb{R}^{N}}\left|\psi_{n}\right|^{2}=1$ and $\left(\frac{-\hbar^{2} \triangle}{2 m} \psi_{n}-\lambda_{n} \psi_{n}\right)$ tends to zero.

Now if we add a Kato potential $V$ to the operator $-\hbar^{2} \triangle / 2 m$, then the domain of 
$\left(-\hbar^{2} \triangle /(2 m)+V\right)$ will not change and the spectrum will still be the half-line: (Corollary (14.10), in [63]),

$$
\sigma_{e s s}\left(\frac{-\hbar^{2} \triangle}{2 m}+V\right)=\sigma_{e s s}\left(\frac{-\hbar^{2} \triangle}{2 m}\right)=[0, \infty)
$$

Example 2 For relativistic Dirac operator, acting square integrable functions on $\mathbb{R}^{3}$, the domain remains the same as in Example 1

$$
D\left(D^{0}\right)=H^{2}\left(\mathbb{R}^{3}\right):=\left\{\psi: \mathbb{R}^{3} \rightarrow \mathbb{C}^{4} \text { such that } \int_{\mathbb{R}^{3}}\left\{|\psi(r)|^{2}+|\triangle \psi(r)|^{2}\right\} d^{3} r \text { is finite }\right\},
$$

where $D^{0}$ is the free Dirac operator defined in Eq. (2.5).

The spectrum of the free Dirac operator is the union of two intervals

$$
\operatorname{Spec}\left(D^{0}\right)=\left(-\infty,-m c^{2}\right] \cup\left[m c^{2}, \infty\right)
$$

The spectrum in Examples 1 and 2 is continuous. If we add an external electric potential $V(r)$, which is smooth and decays at infinity, then the domain $D\left(D^{0}+V\right)$ and the essential spectrum do not change. However, eigenvalues can appear in the gap $\left(-m c^{2}, m c^{2}\right)$.

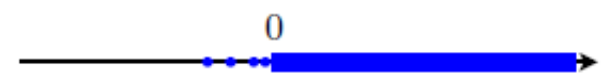

$\operatorname{Spec}\left(-\hbar^{2} \Delta /(2 m)+V(\mathbf{r})\right)$

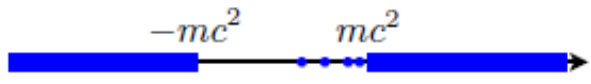

$\operatorname{Spec}\left(D^{0}+V(\mathbf{r})\right)$

Figure 2.1: The typical spectrum of the non-relativistic Schrödinger operator(left), and the Dirac operator with an external potential (right) [3] 


\subsection{Spurious eigenvalue and spurious eigenvectors}

We now recall the definition of spurious eigenvalue, as well as some properties of spurious eigenvectors as in [3].

\section{Definition 2.5.1 (spurious eigenvalues)}

$A$ real number $\lambda \in \mathbb{R}$ is a spurious eigenvalue of the operator $A$, if there exists a sequence of finite dimensional spaces $\left\{V_{n}\right\}_{n \geq 1}$ such that $\left\{V_{n}\right\} \subseteq D(A)$ and $V_{n} \subseteq V_{n+1}$ for all $n$, such as

1. ${\overline{\cup_{n \geq 1} V_{n}}}^{D(A)}=D(A)$;

2. $\lim _{n \rightarrow \infty} \operatorname{dist}\left(\lambda, \sigma\left(A_{\mid V_{n}}\right)\right)=0$;

3. $\lambda \notin \sigma(A)$.

That is: i) the closure of the union of the finite dimensional spaces $V_{n}$ should be equal to the domain of $A$, ii) the limit of the distance between the approximate eigenvalue and the spectrum of $A$ is null, iii) $\lambda$ is not be in the spectrum of the operator $A$. This problem is named spectral pollution and leads to the appearance of spurious states in the approximated spectrum. The spurious states are eigenvalues which do not belong to the spectrum of the continuous operator and which appear in the discretization process. The set of spurious eigenvalues of $A$ is denoted by $\operatorname{Spu}(A)$. The concept of spurious eigenvalues is illustrated, in the following example.

Example 3 We define the Hilbert space $\mathcal{H}=L^{2}(0,2 \pi)$. A function in this space can be expanded using Fourier basis $\{1, \cos (n r), \sin (n r)\}_{n \leq 1}$ as follows:

$$
f(r)=\frac{a_{0}}{\sqrt{2 \pi}}+\frac{1}{\sqrt{\pi}} \sum_{n \geq 1} a_{n} \cos (n r)+b_{n} \sin (n r),
$$

with coefficients defined by:

$$
\begin{gathered}
a_{0}=\frac{1}{\pi} \int_{-\pi}^{\pi} f(x) d x, \\
a_{n}=\frac{1}{\pi} \int_{-\pi}^{\pi} f(x) \cos (n x) d x, \\
b_{n}=\frac{1}{\pi} \int_{-\pi}^{\pi} f(x) \sin (n x) d x .
\end{gathered}
$$


Recall that:

$$
\int_{0}^{2 \pi}|f(r)|^{2} d r=\left|a_{0}\right|^{2}+\sum_{n \geq 1}\left|a_{n}\right|^{2}+\left|b_{n}\right|^{2} .
$$

Now we introduce the orthogonal projection $P$ of $f$ onto the odd modes,

$$
(P f)(r)=\frac{1}{\sqrt{\pi}} \sum_{n \geq 1} b_{n} \sin (n r) .
$$

Operator $P$ is bounded and can be defined in the whole domain $D(P)=L^{2}(0,2 \pi)$, Operator $P$ is diagonal in the Fourier basis, which are thus its eigenvectors. The spectrum $\operatorname{Spec}(P)=\{0,1\}$, is composed of two eigenvalues 0 and 1 having infinite multiplicity. We now construct our approximation space $W_{n}$ by picking all the odd and even functions, which are less than or equal to $n-1$, that is

$W_{n}=\{1, \sin (r), \cos (r), \cdots, \sin ((n-1) r), \cos ((n-1) r), \cos (\theta) \cos (n r)+\sin (\theta) \sin (n r)\}$.

We can better understand spectral pollution by observing this mixture of even and odd modes. Restricting Operator $P$ to the finite sequence $W_{n}$ we get the following $n \times n$ matrix:

$$
P_{\mid W_{n}}=\left(\begin{array}{ccccc}
0 & & & \\
& & & & \\
& 1 & & 0 & \\
& & & & \\
& & & & \\
& 0 & & 1 & \\
& & & & \sin ^{2}(\theta)
\end{array}\right)
$$

and thus the $\operatorname{Spec}(P)_{\mid W_{n}}$ for all $n$, is

$$
\operatorname{Spec}\left(P_{\mid W_{n}}\right)=\left\{0,1, \sin ^{2}(\theta)\right\}
$$

Mixing several modes this way can produce an arbitrary number of spurious modes, which can take any value in the gap $(0,1)$. We find that $\sin ^{2}(\theta)$ is a spurious eigenvalue as it persists, as $n \rightarrow \infty$.

Important notes are:

1. Spurious modes appear in the gap of the essential spectrum. 
2. If the operator is bounded from below or above, spurious modes will never appear below or above the essential spectrum.

In the next example we will describe the eigenvalues that appear in the gap using Rayleigh-Ritz method, but without using any balanced basis, see [65].

Example 4 Consider a self-adjoint operator $T$ defined on a Hilbert space $\mathcal{H}$. Consider an orthogonal projection $P$ from infinite space to finite dimensional space $P: \mathcal{H} \rightarrow \mathcal{L}$, where $\mathcal{L}$ is a finite dimensional subspace of $D(T)$. Now consider the operator $(T f)$ on the Hilbert space $\mathcal{H}=L^{2}(-\pi, \pi)$ defined as follows:

$$
(T f)(x)=\operatorname{sgn}(x) f(x)
$$

where $\operatorname{sgn}(x)=\frac{x}{|x|}$. Since $\|T\|=1$, then $\sigma(T) \subseteq[-1,1]$. but for $\mu \in(-1,1)$, the resolvent operator $(T-\mu \mathbb{I})^{-1}$ is well-defined and bounded, therefore $\sigma(T) \subseteq-1,1$. However, $( \pm 1)$ are eigenvalues of Operator $T$ and belong to $\sigma_{\text {ess }}(T)$. If $\mathcal{L} \subset \mathcal{H}$ is spanned by the set of Fourier basis $\left\{\varphi_{-n}, \varphi_{-n+1}, \cdots, \varphi_{n-1}, \varphi_{n}\right\}$, given by:

$$
\phi_{j}(x)=\frac{1}{\sqrt{2 \pi}} e^{-i j x} j=-n,-n+1, \cdots, n-1, n .
$$

then, the Galerkin approximation applied to $T$ in the finite dimensional subspace $\mathcal{L}$ implies that $\mu_{j}(T, \mathcal{L})$ are the eigenvalue of the $(2 n+1) \times(2 n+1)$ matrix $A$ with entries $(a)_{j k}$ defined as here

$$
a_{j k}=\int_{-\pi}^{\pi} \operatorname{sgn}(x) \phi_{j}(x) \phi_{k}(-x) d x= \begin{cases}0, & \text { if } k-j \text { is even }, \\ \frac{-2 j}{\pi(k-j)}, & \text { if } k-j \text { is odd. }\end{cases}
$$


Matrix $A$ is of the form

$$
A=\left(\begin{array}{cccccc}
0 & N & 0 & N & \cdots & 0 \\
N & 0 & N & 0 & \cdots & N \\
0 & N & 0 & N & \cdots & 0 \\
N & 0 & N & \ddots & \vdots & N \\
\vdots & & & \ddots & \vdots & \\
0 & N & 0 & N & \cdots & 0
\end{array}\right)
$$

here $N$ refers to the different numbers. The matrix $A$ consists of $n+1$ columns (the first set) whose odd entries are zero, and $n$ columns (the second set) whose odd entries are zero. If we disregard the zero entries (which are only $n+1$ entries) in each element of the first set, then we have a linearly dependent set $V=v_{1}, v_{2}, \cdots, v_{n+1}$ where $v_{i} \in \mathbb{R}^{n}, i=1,2, \cdots n+1$. Therefore the columns of the first set of the matrix $A$ is linearly dependent, thus $0 \in \sigma(T)$. As a consequence of the discretization Operator $T$ in finite dimension, we conclude that 0 is a spurious eigenvalue of Operator $T$.

Now we discuss an important property of spurious eigenvectors.

Consider $W_{n}$ a sequence of approximated spaces such that $\lambda \notin \operatorname{Spec}\left(D^{V}\right)$. A solution to the eigenvalue equation can be found as follows:

$$
\left(D^{V}\right)_{\mid W_{n}} x_{n}=\lambda_{n} S_{n} x_{n}
$$

The approximation sequence $W_{n}$ has a spurious eigenvalues $\lambda_{n}$ such that $\lambda_{n} \rightarrow \lambda$ at $n \rightarrow \infty$. Introducing the corresponding approximate eigenfunction

$$
\psi_{n}(r)=\sum_{j=1}^{d_{n}}\left(x_{n}\right)_{j} b_{j}(r)
$$

in $W_{n}$, provided that $\int_{\mathbb{R}^{3}}\left|\psi_{n}(r)\right|^{2} d^{3} r=1$. It means that we have

$$
\int_{\mathbb{R}^{3}} \phi_{n}(r)^{*}\left(D^{0}+V(r)-\lambda_{n}\right) \psi_{n}(r) d^{3} r=0, \forall \phi_{n} \in W_{n}
$$


We recall that by definition, $\psi_{n}$ weakly converge to 0 if $\int_{\mathbb{R}^{3}} \phi_{n}(r)^{*} \psi_{n}(r) d^{3} r \rightarrow 0$ for any $\phi_{n} \in L^{2}\left(\mathbb{R}^{3}\right)$. In other words, it becomes asymptotically orthogonal to any fixed $\phi_{n}$ in the limit $n \rightarrow \infty$. More generally:

Proposition 2.5.1 Spurious eigenvectors tend weakly to 0: If $\lambda \notin \operatorname{Spec}\left(D^{V}\right)$ is a spurious eigenvalue, then the associated eigenvector $\psi_{n} \rightarrow 0$ weakly in $L^{2}\left(\mathbb{R}^{3}\right)$ as $n \rightarrow \infty$.

The idea of the proof as given in [3]: we use the fact that $D^{V}$ is symmetric:

$$
\int_{\mathbb{R}^{3}}\left(\phi_{n}(r)^{*}\left(D^{0}+V(r)-\lambda_{n}\right) \psi_{n}(r) d^{3} r=\left\langle\left(D^{V}-\lambda \mathbb{I}\right) \phi_{n}, \psi_{n}\right\rangle=0\right.
$$

For any $\psi \in H^{1}\left(\mathbb{R}^{3}\right)$ there exists an approximate sequence $\psi_{n} \in W_{n}$, which satisfies the condition:

$$
\lim _{n \rightarrow \infty} \int_{\mathbb{R}^{3}}\left(\left|\psi_{n}(r)-\psi(r)\right|^{2}+\left|\nabla\left(\psi_{n}-\psi\right)(r)\right|^{2}\right) d^{3} r=0
$$

By approximation (2.26) of $W_{n}$ and we can approximate any function $\phi \in H^{1}\left(\mathbb{R}^{3}\right)$, that is we can find a sequence $\phi_{n} \in W_{n}$ such that $D^{V} \phi_{n} \underset{n \rightarrow \infty}{\rightarrow} D^{V} \phi$.

On the other hand, since for all $n, \int\left|\psi_{n}\right|^{2}=1$, we know that $\psi_{n}$ converges weakly to $\psi$ because $W_{n}$ approximate the domain $H^{1}\left(\mathbb{R}^{3}\right)$ of $D^{V}$. Passing to the limit we get $\left\langle\left(D^{V}-\lambda \mathbb{I}\right) \phi, \psi\right\rangle=0$. The last formula is true for all $\phi \in H^{1}\left(\mathbb{R}^{3}\right)$ which implies that $\left(D^{V}-\lambda \mathbb{I}\right) \psi=0$ a.e. As we assume $\lambda \notin \operatorname{Spec}\left(D^{V}\right)$ then we must have $\psi=0$ and this completes the proof.

\subsection{Balanced operator to avoid spectral pollution in Dirac calculation}

Spectral pollution is an important issue that has a long history starting with the celebrated computation of Drake and Goldman [71] in a Slater-type basis set. Several solutions that could assist in avoiding this phenomenon have been proposed in the literature [37,71-77]. In this section, we defined the balanced operator and the strategies that should be used to avoid the spurious modes, when performing the Dirac calculation [3,27]. Throughout this section, we consider $V$ a potential which tends to zero at infinity and the case of $V$ being bounded over the whole space $\mathbb{R}^{3}$, or of 
Coulomb-type. The latter indicates that there are finitely many points $R_{1}, R_{2}, \cdots R_{N}$ (the locations of the nuclei), at which $V$ behaves like:

$$
V(r) \underset{R \rightarrow R_{m}}{\sim}-\frac{\left|e^{2} Z_{m}\right|}{\left|r-R_{m}\right|}, \text { with } 0 \leq e^{2} Z_{m} \leq \frac{\sqrt{3}}{2} \text {. }
$$

and that $V$ is bounded except at $R_{m}$ where $m \in\{1, \cdots N\}$ and (tends to zero at infinity).

According to [27] the reason for our assumption in the case of Coulomb potential when $e^{2} Z_{m} \leq \frac{\sqrt{3}}{2}$ is that, in our units, this correspond to nuclei which have less than 118 protons, which covers all existing atoms. On the other hand, a typical example for which $V \in L^{p}\left(\mathbb{R}^{3}\right) \cup L^{\infty}\left(\mathbb{R}^{3}\right)$ is the case of smeared nuclei $V=\rho \star 1 /|x|$ where $\rho$ is a (sufficiently smooth) distribution of charge for the nuclei.

Furthermore, according to [3], there are two simple motivations in considering general potentials $V(r)$, rather than just $-e^{2} Z / r$. First, the potential of a finiteradius nucleus:

$$
V(r)=e^{2} Z \int_{\mathbb{R}^{3}} \frac{n\left(r^{\prime}\right)}{\left|r-r^{\prime}\right|} d r^{\prime}
$$

is typically bounded, if $n$ is a smooth function. Secondly, in practice, electronic potentials $V(r)$, which will encompass, both the negative nuclear and positive potentials, where the latter is smoother than the one of pointwise nuclei.

In this section also, we are presenting the pollution in balanced basis for the Dirac operator as in [27]. We are studying some methods which allow to avoid pollution by imposing a relation between the vectors of the basis $P H$ and $(1-P) H$ by an operator $L$ which is called a balanced operator.

A symmetric operator $L$ with domain $D(L)$ is a core for the corresponding selfadjoint operator if has the property that its closure is self-adjoint and essentially self-adjoint [63].

More specifically, consider an orthogonal projection $P: H \rightarrow H$ and let $L: P H \rightarrow$ $(1-P) H$ could possibly be unbounded such that:

- $L$ is an injection (1-1): if $L x=0$ for $x \in D(L)$, then $x=0$,

- $D(L) \oplus L D(L)$ is a core of $A$.

Afterwards, the spurious eigenvalue is subsequently defined in the balanced basis [27]. 
Definition 2.6.1 We say that $\lambda \in \mathbb{R}$ is a $(P, L)$-spurious eigenvalue of Operator $A$ if there exists a sequence of finite dimensional spaces $\left(V_{n}^{+}\right)_{n \geq 1}$ with $(V)_{n}^{+} \subseteq V_{n+1}^{+}$for any $n$ where $\left(V_{n}^{+}\right)$refers to the upper part of the spectrum, such that

- ${\overline{\cup_{n \geq 1}\left(V_{n}^{+} \oplus L V_{n}^{+}\right)}}^{D(A)}=D(A)$

- $\lim _{n \rightarrow \infty} \operatorname{dist}\left(\lambda, \sigma\left(A_{\mid V_{n}^{+} \oplus L V_{n}^{+}}\right)\right)=0$,

- $\lambda \notin \sigma(A)$.

We denote by $\operatorname{Spu}(A, P, L)$ the set of $(P, L)$-spurious eigenvalues of the operator $A$ and $\sigma\left(A_{\mid V_{n}^{+} \oplus L V_{n}^{+}}\right)$the set of eigenvalues of the operator $A$ only.

Next we will study the two most used balanced operators in quantum physics and chemistry. The first one is the kinetically balanced operator, while the second one is the atomically balanced operator. 


\subsubsection{Kinetic balance}

Kinetic balance basis function (KBBF), are chosen from Eq. (2.20).

$$
\chi(x) \simeq \frac{1}{2 m c^{2}-V_{c}(x)} \sigma \cdot(-i \nabla) \phi(x)
$$

This suggest to impose this relation between the basis for the upper spinor $\phi$ and the lower spinor $\chi$. Thus this consists of choosing a basis $\left\{\phi_{1}, \cdots, \phi_{n}\right\}$ for the upper spinor and taking the basis $\left\{\sigma \cdot \nabla \phi_{1}, \cdots, \sigma \cdot \nabla \phi_{n}\right\}$ for the lower spinor [71-74].

The kinetic balance operator is defined by

$$
L_{K B}=\frac{1}{2 m c} \sigma \cdot(-i \nabla)
$$

The main result about kinetic balance is the following theorem [27]:

\section{Theorem 2.6.1 (Kinetic balance)}

\section{(i)Bounded potential}

Assume that $V \in L^{p}\left(\mathbb{R}^{3}\right)$ for some $p>3$, such that $\lim _{|x| \rightarrow \infty} V(x)=0$, and

$$
-1+\sup (V)<1+\inf (V),
$$

Then we have

$$
\overline{S p u\left(D^{0}+V, P, L_{K B}\right)}=[-1,-1+\sup (V)] .
$$

\section{(ii)Coulomb potential}

Assume that $-k|x|^{-1}$, where $0<k<\sqrt{3} / 2$, then

$$
\overline{\operatorname{Spu}\left(D^{0}-\frac{k}{|x|}, P, L_{K B}\right)}=[-1,1]
$$

where $P$ is an orthogonal projector.

First, in the case of bounded potentials the theorem states that, spurious eigenvalues are avoided in the upper part of the spectrum, but not necessarily in the lower part. This is due to the kinetic balance condition based on a non relativistic limit for electrons in which the upper spinor is dominant. Secondly, the theorem indicates that for Coulomb potentials, the kinetic balance does not avoid the spurious modes. For the reader interested in the mathematical analysis of the kinetic balance operator, we 
refer to [27]. The strategy used in [27] to prove the existence of spurious modes in the Coulomb case will be discussed shortly in the following section.

\subsubsection{Atomic balance}

Occurrence of spurious modes takes place mainly due to the singularity at $x=0$ of the Coulomb potential. Chemists are rather familiar with this concept [62,78]. Taking into account this singularity amounts to modifying the kinetic balance condition at $x=0$. The atomic balance basis function (ABBF) from Eq. (2.20) becomes

$$
\chi(x) \simeq \frac{1}{2 m c^{2}-V_{c}(x)} \sigma \cdot(-i \nabla) \phi(x)
$$

The atomic balance operator is now defined by:

$$
L_{A B}=\frac{c}{2 m c^{2}-V(x)} \sigma \cdot(-i \nabla)
$$

The main result of atomic balance is the following theorem [27].

\section{Theorem 2.6.2 (Atomic balance)}

Let $V$ be such as $\sup (V)<2,(2-V)^{-2} \nabla V \in L^{\infty}\left(\mathbb{R}^{3}\right)$ and

$$
-\frac{k}{|x|} \leq V(x)
$$

for some $0 \leq k<\sqrt{3} / 2$. We also assume that the positive part $\max (V, 0)$ is in $L^{p}\left(\mathbb{R}^{3}\right)$ for some $p>3$ and that $\lim _{|x| \rightarrow \infty} V(x)=0$. Then we have

$$
\overline{\operatorname{Spu}\left(D^{0}+V, P, L_{A B}\right)}=[-1,-1+\sup V],
$$

where $P$ is orthogonal projector.

The theorem indicates that the atomic balance condition allows us to avoid spurious modes in the upper part of the spectrum even for Coulomb potentials. In particular, the result states that for negative bounded potentials, there will be no pollution, that is the spurious spectrum is empty.

According to these results on Coulomb potentials, spectral pollution can be generated with kinetically balanced basis, but not with atomically balanced basis. As 
we are interested in the two-center system with Coulomb potentials, we will be using the atomic balance basis functions [27].

\section{$2.7 \quad$ B-spline}

In this section, we recall some basic definitions and facts about $B$-splines [4, 79].

A spline function $S$ of degree $k$ consists of a polynomial, $p(x)=a_{0}+a_{1} x+$ $\cdots+a_{k-1} x^{k-1}$ on subintervals $\left[t_{i-1}, t_{i}\right)$ joined together with points, called knots $\left\{t_{i}\right\}$, defined on the real line as:

$$
\ldots<t_{-2}<t_{-1}<t_{0}<t_{1}<t_{2}<\ldots
$$

The $B$-splines of degree $k$ are denoted by $B_{i}^{k}$, where $\{i \in \mathbb{Z}, k \in \mathbb{N}\}$.

$B$-splines are fully determined by the following iterative formula

$$
B_{i}^{k}(x)=\left(\frac{x-t_{i}}{t_{i+k}-t_{i}}\right) B_{i}^{k-1}(x)+\left(\frac{t_{i+k+1}-x}{t_{i+k+1}-t_{i+1}}\right) B_{i+1}^{k-1}(x), \quad(k \geqq 1, \mathrm{i} \in \mathbb{Z}) .
$$

where $x \in\left[t_{i}, t_{i+1}\right)$. $B$-spline can also be written in the following form

$$
B_{i}^{k}(x)=V_{i}^{k}(x) B_{i}^{k-1}(x)+\left(1-V_{i+1}^{k}\right)(x) B_{i+1}^{k-1}(x),
$$

where

$$
V_{i}^{k}(x)=\frac{x-t_{i}}{t_{i+k}-t_{i}}
$$

with initially

$$
\begin{cases}B_{i}^{1}(x)=1, & \text { for } t_{i} \leq x<t_{i+1} \\ B_{i}^{1}(x)=0, & \text { otherwise }\end{cases}
$$

where the points $t_{i}$ are knot coordinates. The number of knots at a given coordinate determines the continuity condition at that point. In the Coulomb singularity position particularly, the number of knot points should be maximal to allow for a discontinuous behavior of the wavefunction $\psi$ at $x=0$. As in [43], thus the number of knot vectors 
$k_{\xi, \eta}$, using and given by the sequences

$$
\begin{gathered}
1=\xi_{1}=\ldots=\xi_{k_{\xi}}<\xi_{k_{\xi}+1}<\ldots<\xi_{n_{\xi}+1}=\ldots=\xi_{n_{\xi}+k_{\xi}}=\xi_{\max } \\
-1=\eta_{1}=\ldots=\eta_{k_{\eta}}<\eta_{k_{\eta}+1}<\ldots<\eta_{n_{\eta}+1}=\ldots=\eta_{n_{\eta}+k_{\eta}}=1
\end{gathered}
$$

Here, $n_{\xi, \eta}$ are the number of spline functions in $\xi$ and $\eta$ coordinates respectively. An explicit representation of $B$-spline of order 3 is given in the following example.

\section{Example 5}

$$
B_{3}(x)= \begin{cases}1 / 2 x^{2}, & 0 \leq x<1 \\ x^{2}+3 x-3 / 2, & 1 \leq x<2, \\ 1 / 2 x^{2}-3 x+9 / 2, & 2 \leq x<3 \\ 0, & \text { otherwise }\end{cases}
$$

on the interval $I=[0,5]$ which can be divided into five subintervals, by the breakpoints $\{0,1,2,3,4,5\}$ joined together with knots vectors $\{0,0,0,1,2,3,4,5,5,5\}$

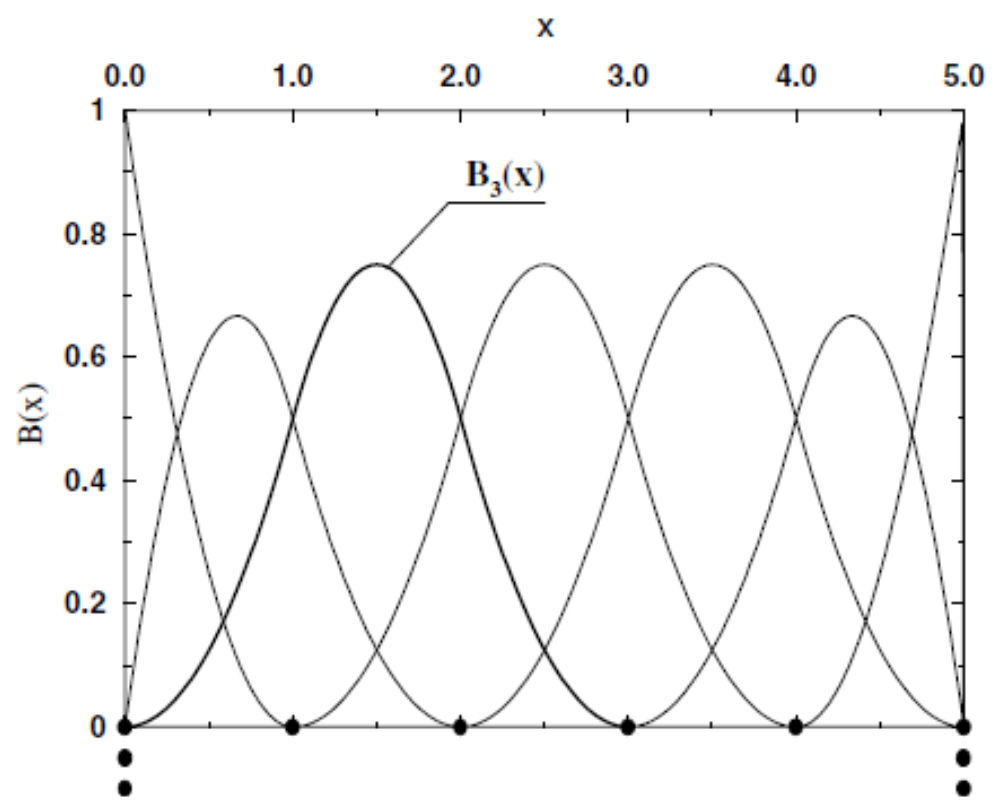

Figure 2.2: B-spline of order $3[4]$ 


\subsubsection{Basic properties of B-splines}

In this subsection, we summarize important properties of $B_{i}^{k}(x)$ for $\{i \in \mathbb{Z}, k \in \mathbb{N}\}$

- $B_{i}^{k}(x)=0$ for $x \notin\left(t_{i}, t_{i+k+1}\right)$, while $B_{i}^{k}(x)>0$ for $x \in\left(t_{i}, t_{i+k+1}\right)$.

- For all $k$ and $x \in\left[t_{0}, t_{n}\right], \sum_{i=-\infty}^{\infty} B_{i}^{k}(x)=1$.

- The set of $B$-splines $\left\{B_{-k}^{k}, B_{-k+1}^{k}, \cdots, B_{0}^{k}\right\}$ are linearly-independent on $\left[t_{0}, t_{n}\right]$.

- For $k \geq 2$,

$$
\frac{d}{d x} B_{i}^{k}(x)=\left(\frac{k}{t_{i+k}-t_{i}}\right) B_{i}^{k-1}(x)+\left(\frac{k}{t_{i+k+1}-t_{i+1}}\right) B_{i+1}^{k-1}(x) .
$$

When $k=1$, the equation is true for all $x$ except $x=t_{i}, t_{i+1}, t_{i+2}$.

- For $k \geq 1$, the $B$-splines belong to the continuity class $C^{k-1}(\mathbb{R})$.

- Integral of $B$-spline satisfies

$$
\int_{-\infty}^{x} B_{i}^{k}(s) d s=\left(\frac{t_{i+k+1}-t_{i}}{k+1}\right) \sum_{j=i}^{\infty} B_{j}^{k+1}(x) .
$$

- For a continuous function $f \in\left[t_{0}, t_{n}\right]$, with a modulus of continuity $\omega(f ; \delta)=$ $\max _{|s-t| \leq \delta}|f(x)-f(t)|$, where $\delta=\max _{-k \leq i \leq n+1}\left|t_{i}-t_{i-1}\right|$, then

$$
\operatorname{dist}\left(f, S_{n}^{k}\right) \leq k^{r} \delta^{r}\left\|f^{(r)}\right\|
$$




\subsubsection{Galerkin method with B-spline basis function for time- independent Dirac equation}

The variational formulation leads to the determination of the stationary points of the Rayleigh-Ritz functional, see Eq. (2.12).

$$
\begin{aligned}
\mathcal{E}[\psi]= & \left\langle\phi \mid\left(V_{c}+m c^{2}\right) \phi\right\rangle_{L^{2}\left(\mathbb{R}^{3}, \mathbb{C}^{2}\right)}+\left\langle R_{0} \phi \mid \chi\right\rangle_{L^{2}\left(\mathbb{R}^{3}, \mathbb{C}^{2}\right)}+\left\langle\chi \mid R_{0} \phi\right\rangle_{L^{2}\left(\mathbb{R}^{3}, \mathbb{C}^{2}\right)} \\
& +\left\langle\chi \mid\left(V_{c}-m c^{2}\right) \chi\right\rangle_{L^{2}\left(\mathbb{R}^{3}, \mathbb{C}^{2}\right)}-E\left[\langle\phi \mid \phi\rangle_{L^{2}\left(\mathbb{R}^{3}, \mathbb{C}^{2}\right)}-\langle\chi \mid \chi\rangle_{L^{2}\left(\mathbb{R}^{3}, \mathbb{C}^{2}\right)}\right]
\end{aligned}
$$

Note that integration by parts was used to write the second term in a convenient form. The notation $\langle\cdot \mid \cdot\rangle_{L^{2}\left(\mathbb{R}^{3}, \mathbb{C}^{2}\right)}$ stands again for the Hermitian inner product on $L^{2}\left(\mathbb{R}^{3}, \mathbb{C}^{2}\right)$ defined for $\Xi$ a two-component spinor, by $\langle\Xi \mid \Xi\rangle=\Xi_{1}^{*} \Xi_{1}+\Xi_{2}^{*} \Xi_{2}$.

In the following section, we define two operators $C$ and $S$ by

$$
\begin{aligned}
C[\psi] & =\int_{\mathbb{R}^{3}}\left[m c^{2}+V_{c}\right]|\phi|^{2}+\left\langle R_{0} \phi \mid \chi\right\rangle+\left\langle\chi \mid R_{0} \phi\right\rangle+\left[V_{c}-m c^{2}\right]|\chi|^{2}, \\
S[\psi] & =\int_{\mathbb{R}^{3}}|\phi|^{2}+|\chi|^{2} .
\end{aligned}
$$

Using the atomically balanced bases, as described in Section 2.6.2, we get the stationary points of $\mathcal{E}$ by setting

$$
\frac{\partial \mathcal{E}}{\partial a_{i}^{(1) *}}=0, \frac{\partial \mathcal{E}}{\partial a_{i}^{(2) *}}=0, \frac{\partial \mathcal{E}}{\partial c_{i}^{(1) *}}=0, \frac{\partial \mathcal{E}}{\partial c_{i}^{(2) *}}=0 .
$$

where $\mathbf{a}=\left[a_{1}^{(1)}, \ldots, a_{N}^{(1)}, a_{1}^{(2)}, \ldots, a_{N}^{(2)}, c_{1}^{(1)}, \ldots, c_{N}^{(1)}, c_{1}^{(2)}, \ldots, c_{N}^{(2)}\right]$ for $i \in[1, \cdots, N]$, are the basis function expansion coefficients. The basis chosen to expand $\psi$ is a $B$-spline basis constructed as follows: first, and as in [43], the large component $\phi$ is expanded as:

$$
\phi_{1,2}(\xi, \eta)=\sum_{n=1}^{N} a_{n}^{(1,2)} B_{n}^{(1,2)}(\xi, \eta)
$$

where $a_{n}^{(1,2)}$ are the coefficients of the basis expansion and $B_{n}^{(1,2)}(\xi, \eta)$ are the basis functions, for components 1 and 2, respectively. Note that this is expressed in the prolate spheroidal coordinate system $\xi, \eta$ described in Eqs. (1.11) and (1.12).

The basis functions can then be written as the tensor product of $B$-spline functions 
as:

$$
B_{n}^{(1,2)}(\xi, \eta)=G^{(1,2)}(\xi, \eta) b_{i}^{k_{\xi}}(\xi) b_{j}^{k_{\eta}}(\eta)
$$

where $n=[i, j] \in \mathbb{Z}^{2}, i \in\left[1, n_{\xi}\right]$ and $j \in\left[1, n_{\eta}\right]$. The overall factor is defined as $[1,44]$

$$
G^{(1,2)}(\xi, \eta)=R\left[\left(\xi^{2}-1\right)\left(1-\eta^{2}\right)\right]^{\frac{\left|\mu_{1,2}\right|}{2}}
$$

This factor accounts for the angular momentum dependence where $\mu_{1,2}:=j_{z} \mp 1 / 2$ (Note that $j_{z}$ is the angular momentum projection on the $z$-axis) and is obtained by considering the boundary condition at $r=0$.

Based on the symmetry of the wavefunction $\psi$, it can be shown that the spinor components would behave like:

$$
\begin{array}{ccc}
\psi_{1,3} & \sim_{r \rightarrow 0} & r^{\left|\mu_{1}\right|} \\
\psi_{2,4} & \sim_{r \rightarrow 0} & r^{\left|\mu_{2}\right|}
\end{array}
$$

The pre-factor $G^{(1,2)}$ explicitly accounts for this dependency. Moreover, it results in well-defined integrals in the Rayleigh-Ritz functional, allowing a better convergence of the method. Using the atomic balance approach, the lower spinor components are then expanded as follows:

$$
\chi=\frac{R_{0}}{2 m c^{2}-V_{c}}\left(\begin{array}{c}
\sum_{n=1}^{N} c_{n}^{(1)} B_{n}^{(1)} \\
\sum_{n=1}^{N} c_{n}^{(2)} B_{n}^{(2)}
\end{array}\right)
$$

which leads in prolate spheroidal coordinates to

$$
\begin{aligned}
& \chi_{1}(\xi, \eta)=\frac{i c}{2 m c^{2}-V_{c}} \sum_{n=1}^{N}\left\{c_{n}^{(2)}\left[-\partial_{r}-\frac{\mu_{2}}{r}\right] B_{n}^{(2)}-c_{n}^{(1)} \partial_{z} B_{n}^{(1)}\right\} \\
& \chi_{2}(\xi, \eta)=\frac{i c}{2 m c^{2}-V_{c}} \sum_{n=1}^{N}\left\{c_{n}^{(1)}\left[-\partial_{r}+\frac{\mu_{1}}{r}\right] B_{n}^{(1)}+c_{n}^{(2)} \partial_{z} B_{n}^{(2)}\right\} .
\end{aligned}
$$

where $r=r(\xi, \eta)$ and $z=(\xi, \eta)$ as in (1.11) and (1.12) for coordinates and in (1.13) for derivatives. 
We obtain the following discrete generalized eigenvalue problem:

$$
\mathbf{C a}=E \mathbf{S a}
$$

where $\mathbf{a}=\left[a_{1}^{(1)}, \ldots, a_{N}^{(1)}, a_{1}^{(2)}, \ldots, a_{N}^{(2)}, c_{1}^{(1)}, \ldots, c_{N}^{(1)}, c_{1}^{(2)}, \ldots, c_{N}^{(2)}\right]$. The matrices of TimeIndependent Dirac Equation are:

$$
\mathbf{C}=\left[\begin{array}{cccc}
\mathbf{C}_{11}^{(1)} & 0 & \mathbf{C}_{11}^{(3)} & \mathbf{C}_{12}^{(3)} \\
0 & \mathbf{C}_{22}^{(1)} & \mathbf{C}_{21}^{(3)} & \mathbf{C}_{22}^{(3)} \\
\mathbf{C}_{11}^{(3) \mathrm{T}} & \mathbf{C}_{21}^{(3) \mathrm{T}} & \mathbf{C}_{11}^{(2)} & \mathbf{C}_{12}^{(2)} \\
\mathbf{C}_{12}^{(3) \mathrm{T}} & \mathbf{C}_{22}^{(3) \mathrm{T}} & \mathbf{C}_{11}^{(2) \mathrm{T}} & \mathbf{C}_{22}^{(2)}
\end{array}\right], \mathbf{S}=\left[\begin{array}{cccc}
\mathbf{S}_{11}^{(1)} & 0 & 0 & 0 \\
0 & \mathbf{S}_{22}^{(1)} & 0 & 0 \\
0 & 0 & \mathbf{S}_{11}^{(2)} & \mathbf{S}_{12}^{(2)} \\
0 & 0 & \mathbf{S}_{12}^{(2) \mathrm{T}} & \mathbf{S}_{22}^{(2)}
\end{array}\right]
$$

The entries of these matrices are defined by:

$$
\begin{aligned}
{\left[\mathbf{C}_{11}^{(1)}\right]_{i j} } & =\int d^{3} x\left\{\left(V_{c}+m c^{2}\right) B_{i}^{(1)} B_{j}^{(1)}\right\} \\
{\left[\mathbf{C}_{22}^{(1)}\right]_{i j} } & =\int d^{3} x\left\{\left(V_{c}+m c^{2}\right) B_{i}^{(2)} B_{j}^{(2)}\right\}
\end{aligned}
$$




$$
\begin{aligned}
& {\left[\mathbf{C}_{11}^{(2)}\right]_{i j}=\int d^{3} x\left\{\left(\partial_{z} B_{i}^{(1)}\right)\left(\partial_{z} B_{j}^{(1)}\right)+\left(\partial_{r} B_{i}^{(1)}\right)\left(\partial_{r} B_{j}^{(1)}\right)\right.} \\
& +\frac{\mu_{1}^{2}}{r^{2}} B_{i}^{(1)} B_{j}^{(1)}-\frac{\mu_{1}}{r} B_{i}^{(1)}\left(\partial_{r} B_{j}^{(1)}\right) \\
& \left.-\frac{\mu_{1}}{r}\left(\partial_{r} B_{i}^{(1)}\right) B_{j}^{(1)}\right\} \times \frac{\left(V_{c}-m c^{2}\right) c^{2}}{\left(2 m c^{2}-V_{c}\right)^{2}} \\
& {\left[\mathbf{C}_{22}^{(2)}\right]_{i j}=\int d^{3} x\left\{\left(\partial_{z} B_{i}^{(2)}\right)\left(\partial_{z} B_{j}^{(2)}\right)+\left(\partial_{r} B_{i}^{(2)}\right)\left(\partial_{r} B_{j}^{(2)}\right)\right.} \\
& +\frac{\mu_{2}^{2}}{r^{2}} B_{i}^{(2)} B_{j}^{(2)}+\frac{\mu_{2}}{r} B_{i}^{(2)}\left(\partial_{r} B_{j}^{(2)}\right) \\
& \left.+\frac{\mu_{2}}{r}\left(\partial_{r} B_{i}^{(2)}\right) B_{j}^{(2)}\right\} \times \frac{\left(V_{c}-m c^{2}\right) c^{2}}{\left(2 m c^{2}-V_{c}\right)^{2}} \\
& {\left[\mathbf{C}_{12}^{(2)}\right]_{i j}=\int d^{3} x\left\{\left(\partial_{z} B_{i}^{(1)}\right)\left(\partial_{r} B_{j}^{(2)}\right)+\frac{\mu_{1}}{r} B_{i}^{(1)}\left(\partial_{z} B_{j}^{(2)}\right)\right.} \\
& \left.-\left(\partial_{r} B_{i}^{(1)}\right)\left(\partial_{z} B_{j}^{(2)}\right)+\frac{\mu_{2}}{r}\left(\partial_{z} B_{i}^{(1)}\right) B_{j}^{(2)}\right\} \\
& \times \frac{\left(V_{c}-m c^{2}\right) c^{2}}{\left(2 m c^{2}-V_{c}\right)^{2}} \\
& {\left[\mathbf{C}_{11}^{(3)}\right]_{i j}=\int d^{3} x\left\{\left(\partial_{z} B_{i}^{(1)}\right)\left(\partial_{z} B_{j}^{(1)}\right)+\left(\partial_{r} B_{i}^{(1)}\right)\left(\partial_{r} B_{j}^{(1)}\right)\right.} \\
& +\frac{\mu_{1}^{2}}{r^{2}} B_{i}^{(1)} B_{j}^{(1)}-\frac{\mu_{1}}{r} B_{i}^{(1)}\left(\partial_{r} B_{j}^{(1)}\right) \\
& \left.-\frac{\mu_{1}}{r}\left(\partial_{r} B_{i}^{(1)}\right) B_{j}^{(1)}\right\} \times \frac{c^{2}}{2 m c^{2}-V_{c}} \\
& {\left[\mathbf{C}_{22}^{(3)}\right]_{i j}=\int d^{3} x\left\{\left(\partial_{z} B_{i}^{(2)}\right)\left(\partial_{z} B_{j}^{(2)}\right)+\left(\partial_{r} B_{i}^{(2)}\right)\left(\partial_{r} B_{j}^{(2)}\right)\right.} \\
& +\frac{\mu_{2}^{2}}{r^{2}} B_{i}^{(2)} B_{j}^{(2)}+\frac{\mu_{2}}{r} B_{i}^{(2)}\left(\partial_{r} B_{j}^{(2)}\right) \\
& \left.+\frac{\mu_{2}}{r}\left(\partial_{r} B_{i}^{(2)}\right) B_{j}^{(2)}\right\} \times \frac{c^{2}}{2 m c^{2}-V_{c}} \\
& {\left[\mathbf{C}_{12}^{(3)}\right]_{i j}=\int d^{3} x\left\{\left(\partial_{z} B_{i}^{(1)}\right)\left(\partial_{r} B_{j}^{(2)}\right)+\frac{\mu_{1}}{r} B_{i}^{(1)}\left(\partial_{z} B_{j}^{(2)}\right)\right.} \\
& \left.-\left(\partial_{r} B_{i}^{(1)}\right)\left(\partial_{z} B_{j}^{(2)}\right)+\frac{\mu_{2}}{r}\left(\partial_{z} B_{i}^{(1)}\right) B_{j}^{(2)}\right\} \times \frac{c^{2}}{2 m c^{2}-V_{c}}
\end{aligned}
$$


then

$$
\begin{aligned}
& {\left[\mathbf{S}_{11}^{(1)}\right]_{i j}=\int d^{3} x\left\{B_{i}^{(1)} B_{j}^{(1)}\right\}=\left[\mathbf{S}_{11}^{(2)}\right]_{i j}} \\
& {\left[\mathbf{S}_{22}^{(1)}\right]_{i j}=\int d^{3} x\left\{B_{i}^{(2)} B_{j}^{(2)}\right\}=\left[\mathbf{S}_{22}^{(2)}\right]_{i j}} \\
& {\left[\mathbf{S}_{11}^{(2)}\right]_{i j}=\int d^{3} x\left\{\left(\partial_{z} B_{i}^{(1)}\right)\left(\partial_{z} B_{j}^{(1)}\right)+\left(\partial_{r} B_{i}^{(1)}\right)\left(\partial_{r} B_{j}^{(1)}\right)\right.} \\
& +\frac{\mu_{1}^{2}}{r^{2}} B_{i}^{(1)} B_{j}^{(1)}-\frac{\mu_{1}}{r} B_{i}^{(1)}\left(\partial_{r} B_{j}^{(1)}\right) \\
& \left.-\frac{\mu_{1}}{r}\left(\partial_{r} B_{i}^{(1)}\right) B_{j}^{(1)}\right\} \times \frac{c^{2}}{\left(2 m c^{2}-V_{c}\right)^{2}} \\
& {\left[\mathbf{S}_{22}^{(2)}\right]_{i j}=\int d^{3} x\left\{\left(\partial_{z} B_{i}^{(2)}\right)\left(\partial_{z} B_{j}^{(2)}\right)+\left(\partial_{r} B_{i}^{(2)}\right)\left(\partial_{r} B_{j}^{(2)}\right)\right.} \\
& +\frac{\mu_{2}^{2}}{r^{2}} B_{i}^{(2)} B_{j}^{(2)}+\frac{\mu_{2}}{r} B_{i}^{(2)}\left(\partial_{r} B_{j}^{(2)}\right) \\
& \left.+\frac{\mu_{2}}{r}\left(\partial_{r} B_{i}^{(2)}\right) B_{j}^{(2)}\right\} \times \frac{c^{2}}{\left(2 m c^{2}-V_{c}\right)^{2}} \\
& {\left[\mathbf{S}_{12}^{(2)}\right]_{i j}=\int d^{3} x\left\{\left(\partial_{z} B_{i}^{(1)}\right)\left(\partial_{r} B_{j}^{(2)}\right)+\frac{\mu_{1}}{r} B_{i}^{(1)}\left(\partial_{z} B_{j}^{(2)}\right)\right.} \\
& \left.-\left(\partial_{r} B_{i}^{(1)}\right)\left(\partial_{z} B_{j}^{(2)}\right)+\frac{\mu_{2}}{r}\left(\partial_{z} B_{i}^{(1)}\right) B_{j}^{(2)}\right\} \\
& \times \frac{c^{2}}{\left(2 m c^{2}-V_{c}\right)^{2}}
\end{aligned}
$$

As we have stated in Section 1.1, the prolate spheroidal coordinates $\xi$ and $\eta$ are related to the cylindrical coordinates $r$ and $z$. The integration measure in prolate spheroidal coordinates is given by

$$
d^{3} x=R^{3}\left(\xi^{2}-\eta^{2}\right) d \xi d \eta d \theta
$$

Note that both matrices $C$ and $S$ are very similar to those obtained using the kinetically-balanced bases [43].

In practice, this linear system is considered a standard eigenvalue solver for sparse matrices. As I mentioned previously in Section 2.6 using kinetic balance basis does not avoid spurious eigenvalue due to the fact that the singularity at $x=0$ in Coulomb 
potential type, justifying the use of atomic balance basis. 


\section{Chapter 3}

\section{Numerical results}

In this chapter we study the convergence of the numerical methods presented in Chapter 2. We will also display the numerical results for two-center problems $\left(\mathrm{H}_{2}^{+}\right.$ and $\left.\mathrm{Th}_{2}^{179+}\right)$.

\subsection{Convergence}

We use $B$-spline basis functions of order 7 which allowes obtain high accuracy. The boundary conditions are chosen as $\phi\left(\xi_{\max }, \eta\right)=0$ and $\chi\left(\xi_{\max }, \eta\right)=0$. We use the number of varieties of elements to obtain precise results after comparing our results with [1] and [2], which are found to be convergent. The tests are the same as those presented in [43]. More specifically, we study and calculate the ground states of dithorium $\left(\mathrm{Th}_{2}^{179+}\right.$ which has $\left.Z_{1,2}=90\right)$ and dihydrogen $\left(\mathrm{H}_{2}^{+}\right.$which has $\left.Z_{1,2}=1\right)$. For dithorium, the semi-inter atomic distance is set to $R=\frac{1}{90} \approx 0.011111$ a.u. while for dihydrogen it is set to $R \approx 1.000$ a.u. On the other hand, the angular momentum is set to $j_{z}=1 / 2$. The results of the calculation of the ground statebinding energy, which is found using $B$-splines (of order 7 ) and other different mesh sizes, are respectively shown in Table 3.1 and 3.2 for $\mathrm{H}_{2}^{+}$and $\mathrm{Th}_{2}^{179+}$. The results presented in these tables show convergence of the method as the number of elements and the B-spline order is increased. The results obtained are relatively accurate, although there is a small difference $\left(\approx 1.86 \times 10^{-8 \%}\right.$ and $\approx 1.01 \times 10^{-4 \%}$ for $\mathrm{H}_{2}^{+}$ and $\mathrm{Th}_{2}^{179+}$, respectively) between our results and the results presented in [1]. The difference can be explained by a different choice of boundary conditions, different 
Table 3.1: Results of the numerical computation for the ground state of $\mathrm{H}_{2}^{+}$for different mesh sizes and B-spline of order 7 . Here, $N_{\xi, \eta}$ is the number of elements found in each coordinates, while $N^{*}$ is the total number of the basis functions utilized. The maximum coordinate was fixed to $\xi_{\max }=30$ a.u. and the angular momentum to $j_{z}=1 / 2$. The calculations are to be compared with the results from [1], where the authors obtained $E_{\mathrm{H}_{2}^{+}}=-1.10264158103$ a.u.

\begin{tabular}{|l|l|l|c|c|c|}
\hline$N_{\xi}$ & $N_{\eta}$ & $N^{*}$ & \multicolumn{2}{|c}{$\begin{array}{c}\mathrm{E}_{\mathrm{H}_{2}^{+}}(\text {a.u. }) \\
\text { Kinetic }\end{array}$} \\
\hline 8 & 8 & 182 & -1.102590816884 & -1.102590816895 & -1.102590816899 \\
10 & 10 & 240 & -1.102638533873 & -1.102638533934 & -1.102638533914 \\
12 & 12 & 306 & -1.102641366239 & -1.102641366228 & -1.102641366222 \\
14 & 14 & 380 & -1.102641554428 & -1.102641554501 & -1.102641554498 \\
16 & 16 & 462 & -1.102641577089 & -1.102641577085 & -1.102641577079 \\
18 & 18 & 552 & -1.102641580210 & -1.102641580229 & -1.102641580219 \\
20 & 20 & 650 & -1.102641580782 & -1.102641580825 & -1.102641580823 \\
\hline
\end{tabular}

Table 3.2: Results of the numerical computation for the ground state of $\mathrm{Th}_{2}^{179+}$ for different mesh sizes and B-spline of order $7 . N_{\xi, \eta}$ is the number of elements existing in each coordinates, while $N^{*}$ is the total number of basis functions utilized. The maximum coordinate was fixed to $\xi_{\max }=15$ a.u. and the angular momentum to $j_{z}=1 / 2$. The calculations are to be compared with the results from [1] and [2], where the authors obtained $E_{\mathrm{Th}_{179}^{+}}=-9504.77424$ a.u and $E_{\mathrm{Th}_{179}^{+}}$ $=-9504.752$ a.u.

\begin{tabular}{|l|l|l|c|c|c|}
\hline$N_{\xi}$ & $N_{\eta}$ & $N^{*}$ & \multicolumn{3}{|c}{$\mathrm{E}_{\mathrm{Th}_{2}^{179+}(\text { a.u. })}$} \\
\hline 8 & 8 & 182 & -9503.998584802 & -9504.592867005 & -9503.999825720 \\
10 & 10 & 240 & -9504.333585765 & -9504.687658554 & -9504.333923392 \\
12 & 12 & 306 & -9504.466070634 & -9504.711111628 & -9504.466246166 \\
14 & 14 & 380 & -9504.539502492 & -9504.722791962 & -9504.539637808 \\
16 & 16 & 462 & -9504.586247153 & -9504.730034585 & -9504.586369144 \\
18 & 18 & 552 & -9504.618392312 & -9504.735005730 & -9504.618508491 \\
20 & 20 & 650 & -9504.641636959 & -9504.738611929 & -9504.641750168 \\
24 & 24 & 870 & -9504.672557123 & -9504.743429586 & -9504.672667124 \\
\hline
\end{tabular}


element formulation and different treatment of the Coulomb singularity.

$$
\gamma_{1,2}=\sqrt{\left(\left|j_{z}\right|+\frac{1}{2}\right)^{2}-\alpha^{2} Z_{1,2}^{2}}
$$

During the ground-state calculation, we have $j_{z}=1 / 2$ and thus $0<\gamma_{1,2}<1$ for $Z_{1,2}<$ 137. Therefore, the wavefunction tends to have a non-integer power-law behavior close to the singularity. The B-spline basis functions, being polynomials with integer powers, they are unable to reproduce exactly this feature. Moreover, we have that

$$
\begin{aligned}
\gamma_{\mathrm{H}} & \approx 0.999947 \text { and } \psi \sim r_{1,2}^{-0.000053} \\
\gamma_{\mathrm{Th}} & \approx 0.568664 \text { and } \psi \sim r_{1,2}^{-0.431336}
\end{aligned}
$$

where $\gamma_{\mathrm{H}, \mathrm{Th}}$ are the gamma associated with a hydrogen or thorium atom. The behavior of the wavefunction $\psi$ is much closer to a power-law for dihydrogen and therefore, it is better reproduced by the B-splines and thus has a faster convergence. One possible strategy that can be used to apply another prefactor in the basis function, which mimics the correct behavior. For instance, it was proposed to multiply the basis functions in (2.46) can be a feasible option, by [1,41,44]

$$
G^{\prime}(\xi, \eta)=r_{1}^{-1+\gamma_{1}} r_{2}^{-1+\gamma_{2}}
$$

with

$$
r_{1}=(\xi+\eta) R, r_{2}=(\xi-\eta) R
$$

where $r_{1,2}$ are the distances from nucleus 1 and 2 . The main problem with this method seems to be the fact that the derivative in the functional becomes singular. To deal with this, a singular coordinate transformation can be performed that allows to transform the singular non-integer behavior near the nuclei to a polynomial approximation $[41,44]$.

In the following section we compute the full spectrum and the results are shown in Tables 3.3 and 3.4 


\subsection{Full spectrum of diatomic molecules}

The spectra are calculated using a mesh of $30 \times 30$ elements. The other parameters are set to the same values as those ones implemented in the previous subsection, where the convergence of the ground state had been discussed. Moreover, the value of the binding energies in the mass gap $\left(\left[-m c^{2}, m c^{2}\right]\right)$ which corresponds to bound states is shifted by $m c^{2}$, by comparison with non-relativistic results. The values in the continua however, are not shifted and are calculated with the Rayleigh-Ritz method only. The result of the dithorium spectrum can be compared to those found in [80]. Both of them are generally in good agreement and compliance, although a small discrepancy can be detected for the higher excited states.

In the Rayleigh-Ritz method, the $n_{\text {binding }}$ bound state energies shown in Tables 3.3 and 3.4 correspond to (the $2 N^{\prime}$ th first eigenvalues) energies in continuam, and $2 N+1$ to $2 N+1+n_{\text {binding }}$ eigenvalues of matrix $\mathbf{C}$. The other eigenvalues can be associated to the "discretized" negative (the $2 N$ 'th first eigenvalues) and positive (the $2 N+2+n_{\text {binding }}$ 'th to the $4 N^{\prime}$ 'th eigenvalues) energy continua.

The convergence of the excited states is very similar to the ground state. The main reason for this is that all the values are approached from above and the order of convergence is close to the one of the ground state. The same technique applies to the states in the positive energy continuum, that is for $E \geq m c^{2}$. Regarding the negative energy states, the convergence occurs from below, however in any other situation, the convergence tends to follow the same trends as the rest of the cases. In addition, the energy values in the continua, particularly the smallest and largest eigenvalues, depend principally on the size of the domain. In each case, the eigenvalues of the positive and negative energy continua accumulate at the points $m c^{2}$ and $-m c^{2}$, respectively.

Our results in Tables 3.3 and 3.4 show also that as expected there are no spurious eigenvalues. 
Table 3.3: Numerical computation of the spectrum of $\mathrm{H}_{2}^{+}$for a mesh size of $30 \times 30$ and B-spline of order 7 . The states of the positive and negative continua are computed with the Rayleigh-Ritz, Min-Max principle, and Atomic Balance methods. The first 5 states are shown.

\begin{tabular}{|l|ll|l|lll|}
\hline $\begin{array}{l}\text { Bound } \\
\text { states }\end{array}$ & \multicolumn{3}{|c}{ Binding energy (a.u.) } & \multicolumn{2}{c|}{$\begin{array}{l}\text { Negative(a.u.) } \\
\text { continuum }\end{array}$} & $\begin{array}{l}\text { Positive(a.u.) } \\
\text { continuum }\end{array}$ \\
\hline 1 & -1.1026413662 & -1.1026415808 & -1.1026415808 & 1 & -18778.95240 & 18778.86549 \\
2 & -0.6675525594 & -0.6675527718 & -0.6675527718 & 2 & -18778.95792 & 18778.86561 \\
3 & -0.4287795568 & -0.4287811584 & -0.4287810919 & 3 & -18778.96471 & 18778.86562 \\
4 & -0.3608697621 & -0.3608710695 & -0.3608690590 & 4 & -18778.97284 & 18778.86741 \\
5 & -0.2554175614 & -0.2554197033 & -0.2553343110 & 5 & -18778.98233 & 18778.86746 \\
\hline
\end{tabular}


Table 3.4: Numerical computation of the spectrum of $\mathrm{Th}_{2}^{179+}$. The mesh size is indicated in the second line and B-splines are of order 7. The atomic balance shown in last column

\begin{tabular}{|l|lll|ll|}
\hline States & Naive RR & & RR & Min-max & Atomic \\
& $14 \times 14$ & $30 \times 30$ & $30 \times 30$ & $16 \times 16$ & $30 \times 30$ \\
\hline 1 & -9504.6525442 & -9504.7243225 & -9504.7475523 & -9504.5862992 & -9504.6416456 \\
2 & -6815.3652913 & -6815.4657298 & -6815.5599111 & -6815.3230307 & -6815.3865298 \\
3 & -4127.8799531 & -4127.8877478 & -4128.1451137 & -4127.8197047 & -4127.8457787 \\
4 & -3374.4958326 & -3374.5117016 & -3374.5143753 & -3374.4569981 & -3374.4767336 \\
5 & -2564.1326367 & -2564.1559253 & -2564.1719708 & -2564.0744037 & -2564.0918230 \\
6 & -2455.9453341 & -2455.9537953 & -2455.9600280 & -2455.8837393 & -2455.9016668 \\
7 & -2010.6579407 & -2010.6535604 & -2010.4321103 & -2010.4241948 & -2010.4261981 \\
8 & -1918.5275474 & -1918.4056980 & -1915.7178408 & -1915.6761267 & -1915.6853488 \\
9 & -1649.5111100 & -1649.2929148 & -1643.9543595 & -1643.9320665 & -1643.9395109 \\
10 & -1349.5529034 & -1344.0855870 & -1313.8071916 & -1313.7606899 & -1313.7699129 \\
11 & -1339.1123032 & -1333.5368147 & -1303.6850950 & -1303.6580541 & -1303.6660492 \\
spurious & -1218.2113620 & -1204.6990945 & & & \\
12 & -1169.3956263 & -1159.1761393 & -1089.6415827 & -1089.6356220 & -1089.6370783 \\
13 & -1138.5709512 & -1131.0151665 & -1084.3699127 & -1084.3519981 & -1084.3522895 \\
14 & -1046.2053120 & -1045.4764538 & -1028.1920826 & -1028.1912423 & -1028.1920249 \\
15 & -1018.4013912 & -984.5252901 & -969.6816867 & -969.64172165 & -969.6482618 \\
\hline
\end{tabular}




\section{Chapter 4}

\section{Time-dependent Dirac equation}

\subsection{Discretization of the time-dependent Dirac equation}

In this section, we discretize the time-dependent Dirac Equation (TDDE), which is defined as:

$$
i \partial_{t} \psi=H \psi,(x, t) \in \mathbb{R}^{3} \times \mathbb{R}_{+}, \quad \psi(x, 0)=\psi_{0}(x), x \in \mathbb{R}^{3}
$$

where $\psi_{0}$ is the initial data and is taken to be a bound state of the time-dependent Dirac operator, that is the eigenfunction associated with the general eigenvalue of $H_{0}$. The TDDE can be written as:

$$
i \partial_{t}\left[\begin{array}{c}
\phi(x, t) \\
\chi(x, t)
\end{array}\right]=\left[\begin{array}{cc}
V_{c}(x)+m c^{2} & R \\
R & V_{c}(x)-m c^{2}
\end{array}\right]\left[\begin{array}{c}
\phi(x, t) \\
\chi(x, t)
\end{array}\right],
$$

where $R:=\boldsymbol{\sigma} \cdot(-i c \nabla-e \mathbf{A}(t))$, and $\sigma$ are the Pauli matrices and $e \mathbf{A}$ is the electromagnetic field:

$$
\begin{aligned}
i \partial_{t}\left[\begin{array}{c}
\phi(x, t) \\
\chi(x, t)
\end{array}\right]= & {\left[\begin{array}{cc}
V_{c}(x)+m c^{2} & R_{0} \\
R_{0} & V_{c}(x)-m c^{2}
\end{array}\right]\left[\begin{array}{c}
\phi(x, t) \\
\chi(x, t)
\end{array}\right] } \\
& +\left[\begin{array}{cc}
0 & -e \boldsymbol{\sigma} \cdot \mathbf{A}(t) \\
-e \boldsymbol{\sigma} \cdot \mathbf{A}(t) & 0
\end{array}\right]\left[\begin{array}{c}
\phi(x, t) \\
\chi(x, t)
\end{array}\right]
\end{aligned}
$$


where $R_{0}=\boldsymbol{\alpha} \cdot(-\mathrm{i} c \nabla)$. We introduce a $B$-spline basis defined using the atomically balanced operator described in 2.6.2, which gives the $j$ 'th basis function spinor as:

$$
\mathcal{B}_{j}:=\left[\begin{array}{c}
\phi_{1, j} \\
\phi_{2, j} \\
\chi_{1, j} \\
\chi_{2, j}
\end{array}\right]=\left[\begin{array}{c}
B_{j}^{(1)} \\
B_{j}^{(2)} \\
\frac{i c}{2 m c^{2}-V_{c}}\left\{\left[-\partial_{r}-\frac{\mu_{2}}{r}\right] B_{j}^{(2)}-\partial_{z} B_{j}^{(1)}\right\} \\
\frac{i c}{2 m c^{2}-V_{c}}\left\{\left[-\partial_{r}+\frac{\mu_{1}}{r}\right] B_{j}^{(1)}+\partial_{z} B_{j}^{(2)}\right\}
\end{array}\right]
$$

where $\mu_{1,2}:=j_{z} \mp 1 / 2$ and $\mathcal{B}_{j}$ are a basis spinor. The weak form of the TDDE is given by:

$$
\left\langle\mathcal{B}_{j} \mid \partial_{t} \psi\right\rangle_{L^{2}\left(\mathbb{R}^{3}, \mathbb{C}^{4}\right)}=\left\langle\mathcal{B}_{j} \mid H \psi\right\rangle_{L^{2}\left(\mathbb{R}^{3}, \mathbb{C}^{4}\right)}, \text { for } j \in[1, N]
$$

We can write this equation as:

$$
\begin{aligned}
\left\langle\phi_{j} \mid \partial_{t} \phi\right\rangle_{L^{2}\left(\mathbb{R}^{3}, \mathbb{C}^{2}\right)}+\left\langle\chi_{j} \mid \partial_{t} \chi\right\rangle_{L^{2}\left(\mathbb{R}^{3}, \mathbb{C}^{2}\right)}= & \left\langle\phi_{j} \mid\left(V_{c}+m c^{2}\right) \phi\right\rangle_{L^{2}\left(\mathbb{R}^{3}, \mathbb{C}^{2}\right)}+\left\langle\chi_{j} \mid\left(V_{c}-m c^{2}\right) \chi\right\rangle_{L^{2}\left(\mathbb{R}^{3}, \mathbb{C}^{2}\right)} \\
& +\left\langle\phi_{j} \mid R_{0} \chi\right\rangle_{L^{2}\left(\mathbb{R}^{3}, \mathbb{C}^{2}\right)}+\left\langle\chi_{j} \mid R_{0} \phi\right\rangle_{L^{2}\left(\mathbb{R}^{3}, \mathbb{C}^{2}\right)} \\
& -e\left\langle\phi_{j} \mid(\boldsymbol{\sigma} \cdot \mathbf{A}) \chi\right\rangle_{L^{2}\left(\mathbb{R}^{3}, \mathbb{C}^{2}\right)}-e\left\langle\chi_{j} \mid(\boldsymbol{\sigma} \cdot \mathbf{A}) \phi\right\rangle_{L^{2}\left(\mathbb{R}^{3}, \mathbb{C}^{2}\right)},
\end{aligned}
$$

for $j \in[1, N]$. These equations are then discretized using the following expansion for the wavefunction as

$$
\begin{aligned}
\phi_{1}(t, \xi, \eta) & =\sum_{n=1}^{N} a_{n}^{(1)}(t) B_{n}^{(1)}(\xi, \eta), \\
\phi_{2}(t, \xi, \eta) & =\sum_{n=1}^{N} a_{n}^{(2)}(t) B_{n}^{(2)}(\xi, \eta) \\
\chi_{1}(t, \xi, \eta) & =\frac{i c}{2 m c^{2}-V_{c}} \sum_{n=1}^{N}\left\{c_{n}^{(2)}(t)\left[-\partial_{r}-\frac{\mu_{2}}{r}\right] B_{n}^{(2)}(\xi, \eta)-c_{n}^{(1)}(t) \partial_{z} B_{n}^{(1)}(\xi, \eta)\right\} \\
\chi_{2}(t, \xi, \eta) & =\frac{i c}{2 m c^{2}-V_{c}} \sum_{n=1}^{N}\left\{c_{n}^{(1)}(t)\left[-\partial_{r}+\frac{\mu_{1}}{r}\right] B_{n}^{(1)}(\xi, \eta)+c_{n}^{(2)}(t) \partial_{z} B_{n}^{(2)}(\xi, \eta)\right\}
\end{aligned}
$$


where $\mu_{1}=j_{z}-1 / 2$ and $\mu_{2}=j_{z}+1 / 2$ (remember that $j_{z}$ is the angular momentum projection on the $z$-axis) $\phi_{1,2}, \chi_{1,2}$ are the large and small components respectively in the atomically balanced basis.

We then obtain a spatial discretization:

$$
i \mathbf{S} \dot{\mathbf{a}}(t)=(\mathbf{C}+\mathbf{D}(t)) \mathbf{a}(t)
$$

with $\mathbf{a}(t)=\left[a_{1}^{(1)}(t), \ldots, a_{n}^{(1)}(t), a_{1}^{(2)}(t), \ldots, a_{n}^{(2)}(t), c_{1}^{(1)}(t), \ldots, c_{n}^{(1)}(t), c_{1}^{(2)}(t), \ldots, c_{n}^{(2)}(t)\right.$, the time dependent unknown.

There are various types of scheme that can be used for the time discretization:

- Explicit Euler scheme:

$$
\mathbf{S a}^{n+1}=\mathbf{S a}^{n}-i \Delta t_{n}\left(\mathbf{C}+\mathbf{D}^{n}\right) \mathbf{a}^{n}
$$

where $\mathbf{a}^{n}=\mathbf{a}\left(t_{n}\right)$ for $n \in N$.

- Semi-implicit scheme (Crank-Nicolson scheme):

$$
\mathbf{S a}^{n+1}=\mathbf{S a}^{n}-i \frac{\Delta t_{n}}{2}\left(\mathbf{C}+\mathbf{D}^{n}\right) \mathbf{a}^{n}-i \frac{\Delta t_{n}}{2}\left(\mathbf{C}+\mathbf{D}^{n+1}\right) \mathbf{a}^{n+1}
$$

- Other time discretizations are naturally possible, such as Runge-Kutta or Simplectic integration schemes [81],

$$
\begin{aligned}
\mathbf{a}\left(t_{f}\right) & =T \exp \left[-i \int_{t_{i}}^{t_{f}} \mathbf{S}^{-1}(\mathbf{C}+\mathbf{D}(t))\right] \mathbf{a}\left(t_{i}\right) \\
& =\exp \left[-i \mathbf{S}^{-1}\left(\mathbf{C}+\mathbf{D}\left(t_{i}+\delta t / 2\right)\right)\right] \mathbf{a}\left(t_{i}\right)+O\left(\delta t^{3}\right)
\end{aligned}
$$

Matrices S, C, are identical to the ones defined above. The only time-dependent matrix $\mathbf{D}(t)$ is the one that includes the electromagnetic field. It is obtained by discretizing in space the following terms of the weak functional:

$$
D(t):=-e\left\langle\phi_{j} \mid(\boldsymbol{\sigma} \cdot \mathbf{A}(t)) \chi\right\rangle_{L^{2}\left(\mathbb{R}^{3}, \mathbb{C}^{2}\right)}-e\left\langle\chi_{j} \mid(\boldsymbol{\sigma} \cdot \mathbf{A}(t)) \phi\right\rangle_{L^{2}\left(\mathbb{R}^{3}, \mathbb{C}^{2}\right)}
$$


By using the basis expansion, it can be written as

$$
\begin{aligned}
& \mathbf{D}=\left[\begin{array}{cccc}
0 & 0 & \mathbf{D}_{11}^{(3)} & \mathbf{D}_{12}^{(3)} \\
0 & 0 & \mathbf{D}_{21}^{(3)} & \mathbf{D}_{22}^{(3)} \\
\mathbf{D}_{11}^{(4)} & \mathbf{D}_{12}^{(4)} & 0 & 0 \\
\mathbf{D}_{21}^{(4)} & \mathbf{D}_{22}^{(4)} & 0 & 0
\end{array}\right] \\
& {\left[\mathbf{D}_{11}^{(3)}\right]_{i j}=i e \int d^{3} x\left\{A_{z} B_{i}^{(1)}\left(\partial_{z} B_{j}^{(1)}\right)-\left(A_{r}-i A_{\theta}\right)\left[-B_{i}^{(1)}\left(\partial_{r} B_{j}^{(1)}\right)+\frac{\mu_{1}}{r} B_{i}^{(1)} B_{j}^{(1)}\right]\right\}} \\
& \times \frac{c}{2 m c^{2}-V_{c}} \\
& {\left[\mathbf{D}_{22}^{(3)}\right]_{i j}=i e \int d^{3} x\left\{A_{z} B_{i}^{(2)}\left(\partial_{z} B_{j}^{(2)}\right)-\left(A_{r}+i A_{\theta}\right)\left[-B_{i}^{(2)}\left(\partial_{r} B_{j}^{(2)}\right)-\frac{\mu_{2}}{r} B_{i}^{(2)} B_{j}^{(2)}\right]\right\}} \\
& \times \frac{c}{2 m c^{2}-V_{c}} \\
& {\left[\mathbf{D}_{12}^{(3)}\right]_{i j}=i e \int d^{3} x\left\{-\left(A_{r}-i A_{\theta}\right) B_{i}^{(1)}\left(\partial_{z} B_{j}^{(2)}\right)-A_{z}\left[-B_{i}^{(1)}\left(\partial_{r} B_{j}^{(2)}\right)-\frac{\mu_{2}}{r} B_{i}^{(1)} B_{j}^{(2)}\right]\right\}} \\
& \times \frac{c}{2 m c^{2}-V_{c}} \\
& {\left[\mathbf{D}_{21}^{(3)}\right]_{i j}=i e \int d^{3} x\left\{\left(A_{r}+i A_{\theta}\right) B_{i}^{(2)}\left(\partial_{z} B_{j}^{(1)}\right)+A_{z}\left[-B_{i}^{(2)}\left(\partial_{r} B_{j}^{(1)}\right)+\frac{\mu_{1}}{r} B_{i}^{(2)} B_{j}^{(1)}\right]\right\}} \\
& \times \frac{c}{2 m c^{2}-V_{c}}
\end{aligned}
$$


and

$$
\begin{array}{rl}
{\left[\mathbf{D}_{11}^{(4)}\right]_{i j}=i e \int d^{3}} & x\left\{-A_{z}\left(\partial_{z} B_{i}^{(1)}\right) B_{j}^{(1)}+\left(A_{r}+i A_{\theta}\right)\left[-\left(\partial_{r} B_{i}^{(1)}\right) B_{j}^{(1)}+\frac{\mu_{1}}{r} B_{i}^{(1)} B_{j}^{(1)}\right]\right\} \\
& \times \frac{c}{2 m c^{2}-V_{c}} \\
{\left[\mathbf{D}_{22}^{(4)}\right]_{i j}=} & i e \int d^{3} x\left\{-A_{z}\left(\partial_{z} B_{i}^{(2)}\right) B_{j}^{(2)}+\left(A_{r}-i A_{\theta}\right)\left[-\left(\partial_{r} B_{i}^{(2)}\right) B_{j}^{(2)}-\frac{\mu_{2}}{r} B_{i}^{(2)} B_{j}^{(2)}\right]\right\} \\
& \times \frac{c}{2 m c^{2}-V_{c}} \\
{\left[\mathbf{D}_{12}^{(4)}\right]_{i j}=} & i e \int d^{3} x\left\{-\left(A_{r}-i A_{\theta}\right)\left(\partial_{z} B_{i}^{(1)}\right) B_{j}^{(2)}-A_{z}\left[-\left(\partial_{r} B_{i}^{(1)}\right) B_{j}^{(2)}+\frac{\mu_{1}}{r} B_{i}^{(1)} B_{j}^{(2)}\right]\right\} \\
& \times \frac{c}{2 m c^{2}-V_{c}} \\
{\left[\mathbf{D}_{21}^{(4)}\right]_{i j}=} & i e \int d^{3} x\left\{\left(A_{r}+i A_{\theta}\right)\left(\partial_{z} B_{i}^{(2)}\right) B_{j}^{(1)}+A_{z}\left[-\left(\partial_{r} B_{i}^{(2)}\right) B_{j}^{(1)}-\frac{\mu_{2}}{r} B_{i}^{(2)} B_{j}^{(1)}\right]\right\} \\
& \times \frac{c}{2 m c^{2}-V_{c}}
\end{array}
$$

A prolate spheroidal coordinates are used to numerically evaluate these integrals. 


\subsection{Mathematical properties}

The semi discrete scheme has several nice mathematical properties which are now detailed.

- $S$ is an Hermitian matrix (and thus has real eigenvalues). Although 0 can be an eigenvalue, it will be necessarily unique, as no spectral pollution is expected with atomically balanced bases [27].

- The use of prolate spheroidal coordinates leads to a very convenient position for local mesh refinement of the molecule nuclei at the corners of the domain.

- One of the main advantages of the proposed approach, comes from the fact that the TDDE solver is consistent with the eigenvalue solver. In particular in the field-free case, we have:

$$
i \partial_{t} \psi=H_{0} \psi, \quad \psi(\cdot, 0)=\phi_{0}(\cdot)
$$

where $H_{0} \phi_{0}=E_{0} \phi_{0}$, with $E_{0}$ the ground state energy, has the following exact solution $\phi_{0}(\cdot) \exp \left(-i E_{0} t\right)$. This property is satisfied at the discrete level up to the order of the time discretization, as the numerical ground state is constructed using the same atomically balanced basis and same mesh as the TDDE solver. Indeed, in that case, $\mathbf{D}$ is identically zero and the semi-discrete scheme is written as, for $t \geq 0$

$$
i \mathbf{S} \dot{a}(t)=\mathbf{C a}(t)
$$

with $\mathbf{a}(0)$, defined by $\mathbf{C a}(0)=E_{0} \mathbf{S a}(0)$. From the explicit scheme:

$$
\mathbf{S a}^{1}=\mathbf{S a}(0)-i \Delta t_{0} \mathbf{C a}(0)=\mathbf{S a}(0)-i \Delta t_{0} E_{0} \mathbf{S a}(0)
$$

That is:

$$
\mathbf{a}^{1}=\left(1-i \Delta t_{0} E_{0}\right) \mathbf{a}(0)
$$


That is by induction and for time steps $\Delta t_{l}$ :

$$
i \mathbf{S} \dot{\mathbf{a}}\left(\sum_{l=0}^{n} \Delta t_{l}\right)=\mathbf{C a}\left(\sum_{l=0}^{n} \Delta t_{l}\right)
$$

Assuming that $\mathbf{a}^{n}=\prod_{l=0}^{n-1}\left(1-i \Delta t_{l} E_{0}\right) \mathbf{a}(0)$ and from

$$
\mathbf{S a}^{n+1}=\left(\mathbf{S}-i \Delta t_{n} \mathbf{C}\right) \mathbf{a}^{n}
$$

we have by induction

$$
\mathbf{a}^{n+1}=\left(1-i \Delta t_{n} E_{0}\right) \mathbf{a}^{n}=\Pi_{l=0}^{n}\left(1-i \Delta t_{l} E_{0}\right) \mathbf{a}(0)
$$

where $\mathbf{a}^{n}=\left[a_{1}^{(1), n}, \ldots, a_{N}^{(1), n}, a_{1}^{(2), n}, \ldots, a_{N}^{(2), n}, c_{1}^{(1), n}, \ldots, c_{N}^{(1), n}, c_{1}^{(2), n}, \ldots, c_{N}^{(2), n}\right]$, which leads to the expected result as

$\Pi_{l=0}^{n}\left(1-i \Delta t_{l} E_{0}\right)=1-i E_{0} \sum_{l=0}^{n} \Delta t_{l}+\mathcal{O}\left(n \Delta t_{\infty}^{2}\right)=\exp \left(-i E_{0} \sum_{l=0}^{n} \Delta t_{l}\right)+\mathcal{O}\left(n \Delta t_{\infty}^{2}\right)$

where $\Delta t_{\infty}=\max _{0 \leq j \leq n} \Delta t_{j}$. This property trivially satisfied is very important from a pratical point of view.

In the case of a semi-implicit (Crank-Nicolson) scheme, we then get

$$
\mathbf{S a}^{1}=\mathbf{S a}(0)-i \frac{\Delta t_{0}}{2} \mathbf{C a}(0)-i \frac{\Delta t_{0}}{2} \mathbf{C a}^{1}
$$

that is

$$
\mathbf{S a}^{1}=\mathbf{S a}(0)-i \frac{\Delta t_{0}}{2} E_{0} \mathbf{S a}(0)-i \frac{\Delta t_{0}}{2} \mathbf{C a}^{1}
$$

then

$$
\left(\mathbf{S}+i \frac{\Delta t_{0}}{2} \mathbf{C}\right) \mathbf{a}^{1}=\mathbf{S}\left(1-i \frac{\Delta t_{0}}{2} E_{0}\right) \mathbf{a}(0)
$$

That is

$$
\mathbf{a}^{1}=\left(1-i \frac{\Delta t_{0}}{2} E_{0}\right)\left(\mathbf{S}+i \frac{\Delta t_{0}}{2} \mathbf{C}\right)^{-1} \mathbf{S a}(0)
$$


Now for $\Delta t_{0}$ small enough

$$
\left(\mathbf{S}+i \frac{\Delta t_{0}}{2} \mathbf{C}\right)^{-1}=\left(\mathbb{I}-i \frac{\Delta t_{0}}{2} \mathbf{S}^{-1} \mathbf{C}-\frac{\Delta t_{0}^{2}}{4}\left(\mathbf{S}^{-1} \mathbf{C}\right)^{2}\right) \mathbf{S}^{-1}+\mathcal{O}\left(\Delta t_{0}^{3}\right)
$$

so that

$$
\mathbf{a}^{1}=e^{-i \Delta t_{0} E_{0}} \mathbf{a}(0)+\mathcal{O}\left(\Delta t_{0}^{3}\right)
$$

By induction, we get

$\mathbf{a}^{n+1}=\Pi_{l=1}^{n}\left[\left(\mathbb{I}+i \frac{\Delta t_{l}}{2} \mathbf{S}^{-1} \mathbf{C}\right)^{-1}\left(\mathbb{I}-i \frac{\Delta t_{l}}{2} \mathbf{S}^{-1} \mathbf{C}\right)\right] \mathbf{a}(0)=e^{-i E_{0} \sum_{l=0}^{n} \Delta t_{l}} \mathbf{a}(0)+\mathcal{O}\left(n \Delta t_{0}^{3}\right)$

and conclude again using similar arguments.

- From above, stability is ensured in the explicit case when the spectral radius of the discrete evolution operator satisfies

$$
\rho\left(\Pi_{l=0}^{n}\left(\mathbb{I}-i \Delta t_{l} \mathbf{S}^{-1}\left(\mathbf{C}+\mathbf{D}^{l}\right)\right)\right) \leq 1
$$

In the field-free case (there is no electromagnetic field), the spectrum was computed using the atomic balanced method. In that case, and as proven by Lewin [17], there is no spurious eigenvalue and all the eigenvalues are also real. We can conclude that, assuming that the eigenvalue solver is exact, the explicit scheme is theoretically unstable. In the semi-implicit case, the scheme writes

$$
\mathbf{S a}^{n+1}=\mathbf{S a}^{n}-i \frac{\Delta t_{n}}{2}\left(\mathbf{C}+\mathbf{D}^{n}\right) \mathbf{a}^{n}-i \frac{\Delta t_{n}}{2}\left(\mathbf{C}+\mathbf{D}^{n+1}\right) \mathbf{a}^{n+1}
$$

so that, we formally have

$$
\mathbf{a}^{n+1}=\Pi_{l=0}^{n}\left(\mathbb{I}+i \frac{\Delta t_{l}}{2} \mathbf{S}^{-1}\left(\mathbf{C}+\mathbf{D}^{l+1}\right)\right)^{-1}\left(\mathbb{I}-i \frac{\Delta t_{l}}{2} \mathbf{S}^{-1}\left(\mathbf{C}+\mathbf{D}^{l}\right)\right) \mathbf{a}^{0}
$$

The requirement for stability is then that

$$
\rho\left(\Pi_{l=0}^{n}\left(\mathbb{I}+i \frac{\Delta t_{l}}{2} \mathbf{S}^{-1}\left(\mathbf{C}+\mathbf{D}^{l+1}\right)\right)^{-1}\left(\mathbb{I}-i \frac{\Delta t_{l}}{2} \mathbf{S}^{-1}\left(\mathbf{C}+\mathbf{D}^{l}\right)\right)\right) \leq 1
$$


Note that in the laser-free case

$$
\begin{gathered}
\rho\left(\prod_{l=0}^{n}\left(\mathbb{I}+i \frac{\Delta t_{l}}{2} \mathbf{S}^{-1} \mathbf{C}\right)^{-1}\left(\mathbb{I}-i \frac{\Delta t_{l}}{2} \mathbf{S}^{-1} \mathbf{C}\right)\right) \\
\leq \prod_{l=0}^{n} \rho\left(\left(\mathbb{I}+i \frac{\Delta t_{l}}{2} \mathbf{S}^{-1} \mathbf{C}\right)^{-1}\left(\mathbb{I}-i \frac{\Delta t_{l}}{2} \mathbf{S}^{-1} \mathbf{C}\right)\right) \\
\leq 1
\end{gathered}
$$

Now as $\mathbf{S}^{-1} \mathbf{C}$ has real eigenvalues, this condition is trivially satisfied and then, as

$$
\rho\left(\left(\mathbb{I}+i \frac{\Delta t_{l}}{2} \mathbf{S}^{-1} \mathbf{C}\right)^{-1}\left(\mathbb{I}-i \frac{\Delta t_{l}}{2} \mathbf{S}^{-1} \mathbf{C}\right)\right)=1
$$

and as a consequence, $\left|\mathbf{a}^{n+1}\right| \leq\left|\mathbf{a}^{0}\right|$, where $\left|\mathbf{a}^{0}\right|$ denotes the $\ell^{2}$ norm of $\mathbf{a}^{0}$, and is dependent of the order of the B-splines which are used. In the laser-field case with electromagnetic field, note that $\mathbf{S}^{-1}\left(\mathbf{C}+\mathbf{D}^{n}\right)$ does not necessarily have real eigenvalues. By regularity of the electromagnetic field, we can however deduce that $\mathbf{D}^{n+1}=\mathbf{D}^{n}+\mathcal{O}\left(\Delta t_{n}\right)$. We can reformulate the problem into

$$
\rho\left(\frac{\overline{\mathbb{I}+i \Delta t \mathbf{A}^{n}}}{\mathbb{I}+i \Delta t \mathbf{A}^{n}+\mathcal{O}\left(\Delta t_{n}^{2}\right)}\right) \leq 1
$$

for some complex matrix $\mathbf{A}^{n}$. The stability condition is ensured up to a $\Delta t_{n}^{2}$ term at each time iteration. For the same reasons as above, the following scheme would then be stable:

$$
\mathbf{S a}^{n+1}=\mathbf{S a}^{n}-i \frac{\Delta t_{n}}{2}\left(\mathbf{C}+\mathbf{D}^{n}\right) \mathbf{a}^{n}-i \frac{\Delta t_{n}}{2}\left(\mathbf{C}+\mathbf{D}^{n}\right) \mathbf{a}^{n+1}
$$

- We assume that for $\psi_{0} \in H$ the solution to TDDE (4.1) formally belongs to $C^{1}(0, T ; V)$, where $V \subseteq L^{2}\left(\mathbb{R}^{3}, \mathbb{C}^{4}\right)$ is an Hilbert space compactly imbedded and dense in $H$ and approximated by a finite dimensional vector space $V_{N}$. We also assume that $\left(\mathcal{B}_{j}\right)_{j}:=\left(\left[B_{j}^{(1,2)}, \chi_{j}^{(1,2)}\right]^{T}\right)_{1 \leq j \leq N}$ is a basis of $V_{N}$, such that ${\overline{V_{N}}}^{V}=V$. Although a full mathematical study of the well-posedness of

$$
i \partial_{t} \psi=H(t) \psi, \quad \psi(0, \cdot)=\psi_{0}(\cdot)
$$


would be necessary to determine $V$ and $H$, we can still give some relevant information about the error estimate without an explicit knowledge of these spaces. We follow the usual procedure, which is for instance presented in [82]. Under the above assumptions we define the canonical projector, $P_{h_{N}}$, from $V$ to $V_{N}$ as follows

$$
P_{h_{N}} \psi\left(t_{n}\right)=\sum_{j=1}^{N} \psi_{j}\left(t_{n}\right) \otimes \mathcal{B}_{j}
$$

with $\mathcal{B}_{j} \in V_{N}$ and

$$
\psi_{j}\left(t_{n}\right)=\left\langle\psi\left(t_{n}, \cdot\right) \mid \mathcal{B}_{j}\right\rangle_{L^{2}\left(\mathbb{R}^{3}, \mathbb{C}^{4}\right)}
$$

The numerical approximation $\psi_{h_{N}}^{n}$ is defined as follows

$$
\psi_{h_{N}}^{n}=\sum_{j=1}^{N} \psi_{h_{N}, j}^{n} \otimes \mathcal{B}_{j}
$$

where

$$
\psi_{h_{N}, j}^{n}=\left[a^{(1,2)}\left(t_{n}\right)_{j}, c^{(1,2)}\left(t_{n}\right)_{j}\right]^{T} \in \mathbb{C}^{4}
$$

and the numerical error:

$$
e_{h_{N}}^{n}:=\psi_{h_{N}}^{n}-P_{h_{N}} \psi\left(t_{n}\right)=\sum_{j=1}^{N}\left(\psi_{h_{N}, j}^{n}-\psi_{j}\left(t_{n}\right)\right) \otimes \mathcal{B}_{j}
$$

We also set:

$$
e_{j}^{n}:=\left\langle e_{h_{N}}^{n} \mid \mathcal{B}_{i}\right\rangle_{\left.L^{2}\left(\mathbb{R}^{3}, \mathbb{C}^{4}\right)\right)}
$$

Now from the scheme

$$
\frac{1}{\Delta t_{n}}\left\langle\psi_{h_{N}}^{n+1}-\psi_{h_{N}}^{n} \mid \mathcal{B}_{j}\right\rangle_{L^{2}\left(\mathbb{R}^{3}, \mathbb{C}^{4}\right)}+\frac{1}{2}\left\langle H\left(t_{n+1}\right) \psi_{h_{N}}^{n+1}-H\left(t_{n}\right) \psi_{h_{N}}^{n} \mid \mathcal{B}_{j}\right\rangle_{L^{2}\left(\mathbb{R}^{3}, \mathbb{C}^{4}\right)}=0
$$


we get

$$
\begin{aligned}
& \frac{1}{\Delta t_{n}}\left\langle\psi_{h_{N}}^{n+1}-P_{h_{N}} \psi\left(t_{n+1}\right) \mid \mathcal{B}_{j}\right\rangle_{L^{2}\left(\mathbb{R}^{3}, \mathbb{C}^{4}\right)}-\frac{1}{\Delta t_{n}}\left\langle\psi_{h_{N}}^{n}-P_{h_{N}} \psi\left(t_{n}\right) \mid \mathcal{B}_{j}\right\rangle_{L^{2}\left(\mathbb{R}^{3}, \mathbb{C}^{4}\right)} \\
& +\frac{1}{2}\left\langle H\left(t_{n+1}\right) \psi_{h_{N}}^{n+1}-H\left(t_{n}\right) \psi_{h_{N}}^{n} \mid \mathcal{B}_{j}\right\rangle_{L^{2}\left(\mathbb{R}^{3}, \mathbb{C}^{4}\right)}=\frac{1}{\Delta t_{n}}\left\langle P_{h_{N}} \psi\left(t_{n}\right)-P_{h_{N}} \psi\left(t_{n+1}\right) \mid \mathcal{B}_{j}\right\rangle_{L^{2}\left(\mathbb{R}^{3}, \mathbb{C}^{4}\right)}
\end{aligned}
$$

which can also be rewritten

$$
\begin{gathered}
\frac{1}{\Delta t_{n}}\left\langle\psi_{h_{N}}^{n+1}-P_{h_{N}} \psi\left(t_{n+1}\right) \mid \mathcal{B}_{j}\right\rangle_{L^{2}\left(\mathbb{R}^{3}, \mathbb{C}^{4}\right)}-\frac{1}{\Delta t_{n}}\left\langle\psi_{h_{N}}^{n}-P_{h_{N}} \psi\left(t_{n}\right) \mid \mathcal{B}_{j}\right\rangle_{L^{2}\left(\mathbb{R}^{3}, \mathbb{C}^{4}\right)} \\
+\frac{1}{2}\left\langle H\left(t_{n+1}\right)\left(\psi_{h_{N}}^{n+1}-P_{h_{N}} \psi\left(t_{n+1}\right)\right) \mid \mathcal{B}_{j}\right\rangle_{L^{2}\left(\mathbb{R}^{3}, \mathbb{C}^{4}\right)}+\frac{1}{2}\left\langle H\left(t_{n}\right)\left(\psi_{h_{N}}^{n}-P_{h_{N}} \psi\left(t_{n}\right)\right) \mid \mathcal{B}_{j}\right\rangle_{L^{2}\left(\mathbb{R}^{3}, \mathbb{C}^{4}\right)} \\
=\frac{1}{\Delta t_{n}}\left\langle P_{h_{N}} \psi\left(t_{n}\right)-P_{h_{N}} \psi\left(t_{n+1}\right) \mid \mathcal{B}_{j}\right\rangle_{L^{2}\left(\mathbb{R}^{3}, \mathbb{C}^{4}\right)}-\frac{1}{2}\left\langle H\left(t_{n+1}\right) P_{h_{N}} \psi\left(t_{n+1}\right)\right. \\
+H\left(t_{n}\right) P_{h_{N}} \psi\left(t_{n}\right)\left|\mathcal{B}_{j}\right\rangle_{L^{2}\left(\mathbb{R}^{3}, \mathbb{C}^{4}\right)}
\end{gathered}
$$

That is

$$
\begin{gathered}
\frac{1}{\Delta t_{n}}\left\langle e_{h_{N}}^{n+1}-e_{h_{N}}^{n} \mid \mathcal{B}_{j}\right\rangle_{L^{2}\left(\mathbb{R}^{3}, \mathbb{C}^{4}\right)}+\frac{1}{2}\left\langle H\left(t_{n+1}\right) e_{h_{N}}^{n+1} \mid \mathcal{B}_{j}\right\rangle_{L^{2}\left(\mathbb{R}^{3}, \mathbb{C}^{4}\right)}+\frac{1}{2}\left\langle H\left(t_{n}\right) e_{h_{N}}^{n} \mid \mathcal{B}_{j}\right\rangle_{L^{2}\left(\mathbb{R}^{3}, \mathbb{C}^{4}\right)} \\
=\frac{1}{\Delta t_{n}}\left\langle P_{h_{N}} \psi\left(t_{n}\right)-P_{h_{N}} \psi\left(t_{n+1}\right) \mid \mathcal{B}_{j}\right\rangle_{L^{2}\left(\mathbb{R}^{3}, \mathbb{C}^{4}\right)} \\
-\frac{1}{2}\left\langle H\left(t_{n+1}\right) P_{h_{N}}\left(t_{n+1}\right)+H\left(t_{n}\right) P_{h_{N}} \psi\left(t_{n}\right) \mid \mathcal{B}_{j}\right\rangle_{L^{2}\left(\mathbb{R}^{3}, \mathbb{C}^{4}\right)}
\end{gathered}
$$

We set

$$
\epsilon_{h_{N}}^{n}:=\frac{1}{\Delta t_{n}}\left(P_{h_{N}} \psi\left(t_{n}\right)-P_{h_{N}} \psi\left(t_{n+1}\right)\right)-\frac{1}{2}\left(H\left(t_{n+1}\right) P_{h_{N}}\left(t_{n+1}\right)+H\left(t_{n}\right) P_{h_{N}} \psi\left(t_{n}\right)\right)
$$

which is also equal to

$$
\begin{aligned}
\left\langle\epsilon_{h_{N}}^{n} \mid \mathcal{B}_{j}\right\rangle_{L^{2}\left(\mathbb{R}^{3}, \mathbb{C}^{4}\right)}= & \left\langle\frac{1}{\Delta t_{n}}\left(P_{h_{N}} \psi\left(t_{n}\right)-P_{h_{N}} \psi\left(t_{n+1}\right)\right)-\frac{1}{2} H\left(t_{n+1}\right)\left(P_{h_{N}} \psi\left(t_{n+1}\right)-\psi\left(t_{n+1}, \cdot\right)\right)\right. \\
& -\frac{1}{2} H\left(t_{n}\right)\left(P_{h_{N}} \psi\left(t_{n}\right)-\psi\left(t_{n}, \cdot\right)\right)+\frac{1}{2} H\left(t_{n+1}\right) \psi\left(t_{n+1}, \cdot\right) \\
& +\frac{1}{2} H\left(t_{n}\right) \psi\left(t_{n}, \cdot\right)\left|\mathcal{B}_{j}\right\rangle_{L^{2}\left(\mathbb{R}^{3}, \mathbb{C}^{4}\right)}
\end{aligned}
$$


From

$$
\frac{d}{d t}\left(P_{h_{N}} \psi\right)=P_{h_{N}} \frac{\partial \psi}{\partial t}
$$

and for all $j$ and all $n \geq 1$

$$
\left\langle\frac{\partial \psi}{\partial t}\left(t_{n}, \cdot\right) \mid \mathcal{B}_{j}\right\rangle_{L^{2}\left(\mathbb{R}^{3}, \mathbb{C}^{4}\right)}=\left\langle H\left(t_{n}\right) \psi\left(t_{n}, \cdot\right) \mid \mathcal{B}_{j}\right\rangle_{L^{2}\left(\mathbb{R}^{3}, \mathbb{C}^{4}\right)}
$$

So that

$$
\begin{aligned}
\left\langle\epsilon_{h_{N}}^{n} \mid \mathcal{B}_{j}\right\rangle_{L^{2}\left(\mathbb{R}^{3}, \mathbb{C}^{4}\right)}= & \left\langle\frac{1}{\Delta t_{n}}\left(P_{h_{N}} \psi\left(t_{n}\right)-P_{h_{N}} \psi\left(t_{n+1}\right)\right)+\frac{1}{2} \frac{\partial \psi}{\partial t}\left(t_{n}, \cdot\right)\right. \\
& +\frac{1}{2} \frac{\partial \psi}{\partial t}\left(t_{n+1}, \cdot\right)-\frac{1}{2} H\left(t_{n+1}\right)\left(P_{h_{N}} \psi\left(t_{n+1}\right)-\psi\left(t_{n+1}, \cdot\right)\right) \\
& -\frac{1}{2} H\left(t_{n}\right)\left(P_{h_{N}} \psi\left(t_{n}\right)-\psi\left(t_{n}, \cdot\right)\right)\left|\mathcal{B}_{j}\right\rangle_{L^{2}\left(\mathbb{R}^{3}, \mathbb{C}^{4}\right)}
\end{aligned}
$$

We now set

$$
\delta_{h_{N}}^{n}:=\frac{1}{\Delta t_{n}}\left(P_{h_{N}} \psi\left(t_{n}\right)-P_{h_{N}} \psi\left(t_{n+1}\right)\right)+\frac{1}{2}\left(\frac{\partial \psi}{\partial t}\left(t_{n}, \cdot\right)+\frac{\partial \psi}{\partial t}\left(t_{n+1}, \cdot\right)\right)
$$

and

$$
\nu_{h_{N}}^{n}=-\frac{1}{2}\left(H\left(t_{n+1}\right)\left(P_{h_{N}} \psi\left(t_{n+1}\right) \psi\left(t_{n+1}, \cdot\right)\right)+H\left(t_{n}\right)\left(P_{h_{N}} \psi\left(t_{n}\right)-\psi\left(t_{n}, \cdot\right)\right)\right)
$$

with $\epsilon_{h_{N}}^{n}=\delta_{h_{N}}^{n}+\nu_{h_{N}}^{n}$. Following [82] and assuming that $\psi \in C^{3}(0, T ; H)$ we get

$$
\left|\delta_{h_{N}}^{n}\right|_{H} \leq \frac{\Delta t_{n}}{8} \int_{t_{n}}^{t_{n+1}}\left|\frac{\partial^{3} \psi}{\partial t^{3}}(s)\right|_{H} d s+\frac{1}{\Delta t_{n}} \int_{t_{n}}^{t_{n+1}}\left|\left(I-P_{h_{N}}\right) \frac{\partial \psi}{\partial t}(s)\right|_{H} d s
$$

Note that $\left\langle\nu_{h_{N}}^{n} \mid \mathcal{B}_{i}\right\rangle_{L^{2}\left(\mathbb{R}^{3}, \mathbb{C}^{4}\right)}$ goes to zero when $h \rightarrow 0$, due to the density of $V_{N}$ is $V$. Details are skipped (at this stage). Finally, from

$$
\begin{aligned}
& \frac{1}{\Delta t_{n}}\left\langle e_{h_{N}}^{n+1}-e_{h_{N}}^{n} \mid \mathcal{B}_{j}\right\rangle_{L^{2}\left(\mathbb{R}^{3}, \mathbb{C}^{4}\right)}+\frac{1}{2}\left\langle H\left(t_{n+1}\right) e_{h_{N}}^{n+1}+H\left(t_{n}\right) e_{h_{N}}^{n} \mid \mathcal{B}_{j}\right\rangle_{L^{2}\left(\mathbb{R}^{3}, \mathbb{C}^{4}\right)}= \\
& \Delta t_{n}\left\langle\delta_{h_{N}}^{n}+\nu_{h_{N}}^{n} \mid \mathcal{B}_{j}\right\rangle_{L^{2}\left(\mathbb{R}^{3}, \mathbb{C}^{4}\right)}
\end{aligned}
$$


we have, without approximation

$$
\mathbf{S e}^{n+1}=\mathbf{S e}^{n}-i \frac{\Delta t_{n}}{2}\left(\mathbf{C}+\mathbf{D}^{n}\right) \mathbf{e}^{n}-i \frac{\Delta t_{n}}{2}\left(\mathbf{C}+\mathbf{D}^{n+1}\right) \mathbf{e}^{n}+\Delta t_{n}\left(\delta^{n}+\nu^{n}\right)
$$

where

$$
\begin{aligned}
\mathbf{e}^{n}= & {\left[\psi_{1}^{(1)}\left(t_{n}\right)-a_{1}^{(1), n}, \ldots, \psi_{h_{N}}^{(1)}\left(t_{n}\right)-a_{N}^{(1), n}, \psi_{1}^{(2)}\left(t_{n}\right)-a_{1}^{(2), n}, \ldots, \psi_{h_{N}}^{(2)}\left(t_{n}\right)-a_{N}^{(2), n},\right.} \\
& \left.\psi_{1}^{(3)}\left(t_{n}\right)-c_{1}^{(1), n}, \ldots, \psi_{h_{N}}^{(3)}\left(t_{n}\right)-c_{N}^{(1), n}, \psi_{1}^{(4)}\left(t_{n}\right)-c_{1}^{(2), n}, \ldots, \psi_{h_{N}}^{(4)}\left(t_{n}\right)-c_{N}^{(2), n}\right]
\end{aligned}
$$

and $\delta^{n}=\left(\left\langle\delta_{h_{N}}^{n} \mid \mathcal{B}_{j}\right\rangle_{L^{2}\left(\mathbb{R}^{3}, \mathbb{C}^{4}\right)}\right)_{j}, \nu^{n}=\left(\left\langle\nu_{h_{N}}^{n} \mid \mathcal{B}_{j}\right\rangle_{L^{2}\left(\mathbb{R}^{3}, \mathbb{C}^{4}\right)}\right)_{j}$. Now we deduce

$$
\left(\mathbf{S}+i \frac{\Delta t_{n}}{2}\left(\mathbf{C}+\mathbf{D}^{n+1}\right)\right) \mathbf{e}^{n+1}=\left(\mathbf{S}-i \frac{\Delta t_{n}}{2}\left(\mathbf{C}+\mathbf{D}^{n}\right)\right) \mathbf{e}^{n}+\Delta t_{n}\left(\delta^{n}+\nu^{n}\right)
$$

Then

$$
\begin{aligned}
\mathbf{e}^{n+1}= & \left(\mathbf{S}+i \frac{\Delta t_{l}}{2}\left(\mathbf{C}+\mathbf{D}^{l+1}\right)\right)^{-1}\left(\mathbf{S}-i \frac{\Delta t_{l}}{2}\left(\mathbf{C}+\mathbf{D}^{l}\right)\right) \mathbf{e}^{n} \\
& +\Delta t_{n}\left(\mathbf{S}+i \frac{\Delta t_{l}}{2}\left(\mathbf{C}+\mathbf{D}^{n+1}\right)\right)^{-1}\left(\delta^{n}+\nu^{n}\right)
\end{aligned}
$$

Finally from

$$
\left|\psi_{h_{N}}^{n}-\psi\left(t_{n}, \cdot\right)\right|_{H} \leq\left|\psi_{h_{N}}^{n}-P_{h_{N}} \psi\left(t_{n}\right)\right|_{H}+\left|\left(I-P_{h_{N}}\right) \psi\left(t_{n}, \cdot\right)\right|_{H}
$$

Under the strong but reasonable assumptions for TDDE (4.1) that made at the beginning of this paragraph, we can formally conclude of the convergence of the method. 


\section{Chapter 5}

\section{Conclusion}

In this thesis, we studied some computational methods for solving the Dirac Equation for both time-independent and time-dependent cases. In this purpose, a Galerkin method is applied using B-spline basis function with prolate spheroidal coordinates. This choice of coordinate system is very convenient for the numerical implementation because the Coulomb singularities are located in domain boundaries. In particular we investigated the numerical convergence of Galerkin techniques combined with atomically balanced operator which allows us to avoid spectral pollution for the Dirac operator. Specifically, we studied and calculated the ground and excited states of two-center problems (molecules $\mathrm{H}_{2}^{+}$and $\mathrm{Th}_{2}^{197+}$ ). We use the number of different elements to obtain accurate results and we found that our results are very closed to those in the literature. High order B-spline basis functions were also used to obtain high accuracy. We computed the full spectrum of $\mathrm{H}_{2}^{+}$and $T h_{2}^{179+}$ using a mesh of $30 \times 30$ elements. We concluded that, there were no spurious eigenvalue in these numerical results. The solution obtained from these methods can be used as an initial data and is taken to be a Cauchy data of the time dependent Dirac equation (TDDE). In this equation Galerkin methods combined with atomically balanced operator are used to determine the time dependent unknown coefficients. The numerical methods for solving the TDDE have already been implemented on a high performance computer and will be tested in the new future. 


\section{List of References}

[1] O. Kullie, D. Kolb, and A. Rutkowski, "Two-spinor fully relativistic finiteelement (fem) solution of the two-center Coulomb problem," Chemical physics letters, vol. 383, no. 3, pp. 215-221, 2004.

[2] A. Artemyev, A. Surzhykov, P. Indelicato, G. Plunien, and T. Stöhlker, "Finite basis set approach to the two-centre Dirac problem in cassini coordinates," Journal of Physics B: Atomic, Molecular and Optical Physics, vol. 43, no. 23, p. 235207, 2010.

[3] M. Lewin and E. Séré, "Spurious modes in Dirac calculations and how to avoid them," arXiv preprint arXiv:1306.5401, 2013.

[4] H. Bachau, E. Cormier, P. Decleva, J. Hansen, and F. Martin, "Applications of B-splines in atomic and molecular physics," Reports on Progress in Physics, vol. 64, no. 12, p. 1815, 2001.

[5] G. A. Mourou, T. Tajima, and S. V. Bulanov, "Optics in the relativistic regime," Reviews of Modern Physics, vol. 78, no. 2, p. 309, 2006.

[6] A. Di Piazza, C. Müller, K. Hatsagortsyan, and C. Keitel, "Extremely highintensity laser interactions with fundamental quantum systems," Reviews of Modern Physics, vol. 84, no. 3, p. 1177, 2012.

[7] J. Reinhardt, B. Müller, and W. Greiner, "Theory of positron production in heavy-ion collisions," Physical Review A, vol. 24, no. 1, p. 103, 1981.

[8] G. R. Mocken and C. H. Keitel, "Fft-split-operator code for solving the Dirac equation in 2+ 1 dimensions," Computer Physics Communications, vol. 178, no. 11, pp. 868-882, 2008.

[9] G. R. Mocken and C. H. Keitel, "Quantum dynamics of relativistic electrons," Journal of Computational Physics, vol. 199, no. 2, pp. 558-588, 2004.

[10] G. R. Mocken and C. H. Keitel, "Fft-split-operator code for solving the Dirac equation in $2+1$ dimensions," Computer Physics Communications, vol. 178, no. 11 , pp. $868-882,2008$.

[11] J. W. Braun, Q. Su, and R. Grobe, "Numerical approach to solve the timedependent Dirac equation," Phys. Rev. A, vol. 59, pp. 604-612, Jan 1999. 
[12] Z. Huang, S. Jin, P. A. Markowich, C. Sparber, and C. Zheng, "A time-splitting spectral scheme for the Maxwell-Dirac system," J. Comput. Phys., vol. 208, no. 2, pp. 761-789, 2005.

[13] W. Bao and X.-G. Li, "An efficient and stable numerical method for the MaxwellDirac system," J. Comput. Phys., vol. 199, no. 2, pp. 663-687, 2004.

[14] S. Succi and R. Benzi, "Lattice boltzmann equation for quantum mechanics," Physica D: Nonlinear Phenomena, vol. 69, no. 34, pp. 327 - 332, 1993.

[15] E. Lorin and A. Bandrauk, "A simple and accurate mixed P0-Q1 solver for the maxwell-Dirac equations," Nonlinear Analysis: Real World Applications, vol. 12, no. 1, pp. 190-202, 2011.

[16] F. Fillion-Gourdeau, E. Lorin, and A. D. Bandrauk, "Numerical solution of the time-dependent Dirac equation in coordinate space without fermion-doubling," Computer Physics Communications, vol. 183, no. 7, pp. 1403-1415, 2012.

[17] F. Fillion-Gourdeau, E. Lorin, and A. D. Bandrauk, "A split-step numerical method for the time-dependent Dirac equation in 3-d axisymmetric geometry," to Apear in J. Comput. Phys., 2014.

[18] G. R. Mocken and C. H. Keitel, "Ff-split operator code for solving the Dirac equation in $2+1$ dimensions," Comput. Phys. Comm., vol. 178, no. 11, pp. 868$882,2008$.

[19] E. Ackad and M. Horbatsch, "Numerical solution of the Dirac equation by a mapped fourier grid method," Journal of Physics A: Mathematical and General, vol. 38, no. 14, p. 3157, 2005.

[20] E. Ackad and M. Horbatsch, "Calculation of electron-positron production in supercritical uranium-uranium collisions near the Coulomb barrier," Phys. Rev. $A$, vol. 78, p. 062711, Dec 2008.

[21] S. Salomonson and P. Öster, "Relativistic all-order pair functions from a discretized single-particle Dirac Hamiltonian," Phys. Rev. A, vol. 40, pp. 5548-5558, Nov 1989.

[22] S. Salomonson and P. Öster, "Solution of the pair equation using a finite discrete spectrum," Phys. Rev. A, vol. 40, pp. 5559-5567, Nov 1989.

[23] S. Selstø, E. Lindroth, and J. Bengtsson, "Solution of the Dirac equation for hydrogenlike systems exposed to intense electromagnetic pulses," Phys. Rev. A, vol. 79, p. 043418, Apr 2009.

[24] F. Gelis, K. Kajantie, and T. Lappi, "Quark-antiquark production from classical fields in heavy-ion collisions: $1+1$ dimensions," Phys. Rev. C, vol. 71, p. 024904, Feb 2005.

[25] U. Becker, N. Grun, and W. Scheid, "Solution of the time-dependent Dirac equation by the finite difference method and application for $\mathrm{Ca}^{20+}+\mathrm{U}^{91+}$," Journal of Physics B: Atomic and Molecular Physics, vol. 16, no. 11, p. 1967, 1983. 
[26] W. Kutzelnigg, "Basis set expansion of the Dirac operator without variational collapse," International Journal of Quantum Chemistry, vol. 25, no. 1, pp. 107129, 1984.

[27] M. Lewin and É. Séré, "Spectral pollution and how to avoid it (with applications to Dirac and periodic Schrödinger operators)," Proc. Lond. Math. Soc. (3), vol. 100, no. 3, pp. 864-900, 2010.

[28] J. D. Talman, "Minimax principle for the Dirac equation," Phys. Rev. Lett., vol. 57, pp. 1091-1094, Sep 1986.

[29] J. Dolbeault, M. J. Esteban, and E. Séré, "Variational characterization for eigenvalues of Dirac operators," Calculus of Variations and Partial Differential Equations, vol. 10, pp. 321-347, 2000.

[30] J. Dolbeault, M. J. Esteban, and E. Séré, "On the eigenvalues of operators with gaps. application to Dirac operators," Journal of Functional Analysis, vol. 174, no. 1, pp. $208-226,2000$.

[31] J. Dolbeault, M. J. Esteban, and E. Séré, "A variational method for relativistic computations in atomic and molecular physics," International Journal of Quantum Chemistry, vol. 93, pp. 149 - 155, 2003.

[32] H. Wallmeier and W. Kutzelnigg, "Basis-set expansion of the Dirac equation without variational collapse: Numerical test of the forth-back free-particle foldywouthuysen transformation," Phys. Rev. A, vol. 28, pp. 3092-3094, Nov 1983.

[33] H. M. Quiney, I. P. Grant, and S. Wilson, "The Dirac equation in the algebraic approximation," Physica Scripta, vol. 36, no. 3, p. 460, 1987.

[34] I. P. Grant and H. M. Quiney, "Rayleigh-Ritz approximation of the Dirac operator in atomic and molecular physics," Phys. Rev. A, vol. 62, p. 022508, Jul 2000 .

[35] R. E. Stanton and S. Havriliak, "Kinetic balance: A partial solution to the problem of variational safety in Dirac calculations," The Journal of chemical physics, vol. 81, no. 4, pp. 1910-1918, 1984.

[36] L. Visscher, P. J. C. Aerts, O. Visser, and W. C. Nieuwpoort, "Kinetic balance in contracted basis sets for relativistic calculations," International Journal of Quantum Chemistry, vol. 40, no. S25, pp. 131-139, 1991.

[37] V. M. Shabaev, I. I. Tupitsyn, V. A. Yerokhin, G. Plunien, and G. Soff, "Dual kinetic balance approach to basis-set expansions for the Dirac equation," Phys. Rev. Lett., vol. 93, p. 130405, Sep 2004.

[38] Y. V. Vanne and A. Saenz, "Solution of the time-dependent Dirac equation for multiphoton ionization of highly charged hydrogenlike ions," Phys. Rev. A, vol. 85, p. 033411, Mar 2012. 
[39] S. R. McConnell, A. N. Artemyev, M. Mai, and A. Surzhykov, "Solution of the two-center time-dependent Dirac equation in spherical coordinates: Application of the multipole expansion of the electron-nuclei interaction," Phys. Rev. A, vol. 86, p. 052705, Nov 2012.

[40] C. F. Fischer and O. Zatsarinny, "A B-spline galerkin method for the Dirac equation," Computer Physics Communications, vol. 180, no. 6, pp. 879 - 886, 2009.

[41] L. Yang, D. Heinemann, and D. Kolb, "Fully numerical relativistic calculations for diatomic molecules using the finite-element method," Physical Review A, vol. 48, no. 4, p. 2700, 1993.

[42] L. Laaksonen and I. P. Grant, "Two-dimensional fully numerical solutions of molecular Dirac equations. one-electron molecules," Chemical Physics Letters, vol. 109, no. 5, pp. $485-487,1984$.

[43] F. Fillion-Gourdeau, E. Lorin, and A. Bandrauk, "Numerical solution of the timeindependent Dirac equation for diatomic molecules: B-splines without spurious states," Physical Review A - Atomic, Molecular, and Optical Physics, vol. 85, no. 2, 2012. cited By (since 1996)1.

[44] O. Kullie, C. Düsterhöft, and D. Kolb, "Dirac-fock finite element method (fem) calculations for some diatomic molecules," Chemical physics letters, vol. 314, no. 3, pp. 307-310, 1999.

[45] D. Sundholm, "Fully numerical solutions of molecular Dirac equations for highly charged one-electron homonuclear diatomic molecules," Chemical Physics Letters, vol. 223, no. 5-6, pp. 469 - 473, 1994.

[46] D. Sundholm, P. Pyykkö, and L. Laaksonen, "Two-dimensional, fully numerical solutions of second-order Dirac equations for diatomic molecules. part 3," Physica Scripta, vol. 36, no. 3, p. 400, 1987.

[47] F. Fillion-Gourdeau, E. L. de la Grandmaison, and A. D. Bandrauk, "Relativistic ground state of diatomic molecules from the numerical solution of the Dirac equation on parallel computers," in Journal of Physics: Conference Series, vol. 341, p. 012006, IOP Publishing, 2012.

[48] B. Mueller, J. Rafelski, and W. Greiner, "Solution of the Dirac equation with two Coulomb centres," Physics Letters B, vol. 47, no. 1, pp. 5-7, 1973.

[49] R. Dautray and J.-L. Lions, Mathematical Analysis and Numerical Methods for Science and Technology: Volume 3 Spectral Theory and Applications, vol. 3. Springer, 2000.

[50] P. Schluter, K.-H. Wietschorke, and W. Greiner, "The Dirac equation in orthogonal coordinate systems. i. the local representation," Journal of Physics A: Mathematical and General, vol. 16, no. 9, p. 1999, 1983.

[51] M. Aubert, N. Bessis, and G. Bessis, "Prolate-spheroidal orbitals for homonuclear and heteronuclear diatomic molecules. i. basic procedure," Physical Review A, vol. 10, no. 1, p. 51, 1974. 
[52] A. D. Becke, "Numerical hartree-fock-slater calculations on diatomic molecules," The Journal of Chemical Physics, vol. 76, no. 12, pp. 6037-6045, 1982.

[53] J. Kobus, L. Laaksonen, and D. Sundholm, "A numerical Hartree-Fock program for diatomic molecules," Computer physics communications, vol. 98, no. 3, pp. 346-358, 1996.

[54] L. Boulton and M. Levitin, "On approximation of the eigenvalues of perturbed periodic Schrödinger operators," Journal of Physics A: Mathematical and Theoretical, vol. 40, no. 31, p. 9319, 2007.

[55] E. Cancès, V. Ehrlacher, and Y. Maday, "Periodic Schrödinger operators with local defects and spectral pollution," SIAM Journal on Numerical Analysis, vol. 50, no. 6, pp. 3016-3035, 2012.

[56] Z. J. Csendes and P. Silvester, "Numerical solution of dielectric loaded waveguides: I-finite-element analysis," Microwave Theory and Techniques, IEEE Transactions on, vol. 18, no. 12, pp. 1124-1131, 1970.

[57] A. Bossavit, "Solving Maxwell equations in a closed cavity, and the question ofspurious modes'," Magnetics, IEEE Transactions on, vol. 26, no. 2, pp. 702705,1990 .

[58] W. Schroeder and I. Wolff, "The origin of spurious modes in numerical solutions of electromagnetic field eigenvalue problems," Microwave Theory and Techniques, IEEE Transactions on, vol. 42, no. 4, pp. 644-653, 1994.

[59] J. Rappaz, J. S. Hubert, E. S. Palencia, and D. Vassiliev, "On spectral pollution in the finite element approximation of thin elastic membrane shells," Numerische Mathematik, vol. 75, no. 4, pp. 473-500, 1997.

[60] C. F. Bunting and W. A. Davis, "A functional for dynamic finite-element solutions in electromagnetics," Antennas and Propagation, IEEE Transactions on, vol. 47, no. 1, pp. 149-156, 1999.

[61] D. Boffi, F. Brezzi, and L. Gastaldi, "On the problem of spurious eigenvalues in the approximation of linear elliptic problems in mixed form," Mathematics of Computation of the American Mathematical Society, vol. 69, no. 229, pp. 121$140,2000$.

[62] P. Fernandes and M. Raffetto, "Counterexamples to the currently accepted explanation for spurious modes and necessary and sufficient conditions to avoid them," Magnetics, IEEE Transactions on, vol. 38, no. 2, pp. 653-656, 2002.

[63] S. Hislop, Introduction to Spectral Theory. Springer, 1996.

[64] A. Quarteroni, R. Sacco, and F. Saleri, "Two-point boundary value problems," Numerical Mathematics, pp. 539-587, 2007.

[65] H. Almanasreh, The Dirac Equation: Numerical and Asymptotic Analysis. PhD thesis, Chalmers University of Technology, 2012. 
[66] I. P. Grant, Relativistic Quantum Theory of Atoms and Molecules: Theory and Computation. Springer Series on Atomic, Optical, and Plasma Physics, Springer, 2006 .

[67] S. N. Datta and G. Devaiah, "The minimax technique in relativistic hartree-fock calculations," Pramana, vol. 30, no. 5, pp. 387-405, 1988.

[68] M. J. Esteban and E. Séré, "Existence and multiplicity of solutions for linear and nonlinear Dirac problems," Partial differential equations and their applications, vol. 12, p. 107, 1997.

[69] C. Itzykson and J. B. Zuber, Quantum Field Theory. Mcgraw-hill, 1980.

[70] B. Thaller, The Dirac equation, vol. 31. Springer-Verlag Berlin, 1992.

[71] G. W. Drake and S. Goldman, "Application of discrete-basis-set methods to the Dirac equation," Physical Review A, vol. 23, no. 5, p. 2093, 1981.

[72] I. P. Grant, "Conditions for convergence of variational solutions of Dirac's equation in a finite basis," Phys. Rev. A, vol. 25, pp. 1230-1232, Feb 1982.

[73] W. Kutzelnigg, "Basis set expansion of the Dirac operator without variational collapse," International Journal of Quantum Chemistry, vol. 25, no. 1, pp. 107129, 1984.

[74] R. E. Stanton and S. Havriliak, "Kinetic balance: A partial solution to the problem of variational safety in Dirac calculations," The Journal of chemical physics, vol. 81, no. 4, pp. 1910-1918, 1984.

[75] K. G. Dyall and K. Fægri Jr, "Kinetic balance and variational bounds failure in the solution of the Dirac equation in a finite gaussian basis set," Chemical Physics Letters, vol. 174, no. 1, pp. 25-32, 1990.

[76] G. Pestka, "Spurious roots in the algebraic Dirac equation," Physica Scripta, vol. 68 , no. 4, p. 254, 2003.

[77] L. Boulton and N. Boussaid, "Non-variational computation of the eigenstates of Dirac operators with radially symmetric potentials," LMS Journal of Computation and Mathematics, vol. 13, pp. 10-32, 2010.

[78] B. Klahn and W. A. Bingel, "The convergence of the Rayleigh-Ritz method in quantum chemistry," Theoretica chimica acta, vol. 44, no. 1, pp. 27-43, 1977.

[79] D. Kincaid and W. Cheney, Mathematics of Scientific Computing. Pacific Grove, CA: Brooks/Cole, 1996.

[80] I. Grant, "B-spline methods for radial Dirac equations," Journal of Physics B: Atomic, Molecular and Optical Physics, vol. 42, no. 5, p. 055002, 2009.

[81] P. J. van der Houwen and B. P. Sommeijer, "Explicit Runge-Kutta (-nyström) methods with reduced phase errors for computing oscillating solutions," SIAM Journal on Numerical Analysis, vol. 24, no. 3, pp. 595-617, 1987. 
[82] P.-A. Raviart and J.-M. Thomas, Introduction à l'analyse numérique des équations aux dérivées partielles. Masson, 1983. 